\title{
Treatment of organic pollutants by homogeneous and heterogeneous Fenton reaction processes
}

\author{
Bhawana Jain $^{1} \cdot$ Ajaya Kumar Singh $^{1} \cdot$ Hyunook Kim $^{2} \cdot$ Eric Lichtfouse $^{3} \cdot$ Virender K. Sharma $^{4}$
}

\begin{abstract}
Nowadays, the water ecosystem is being polluted due to the rapid industrialization and massive use of antibiotics, fertilizers, cosmetics, paints, and other chemicals. Chemical oxidation is one of the most applied processes to degrade contaminants in water. However, chemicals are often unable to completely mineralize the pollutants. Enhanced pollutant degradation can be achieved by Fenton reaction and related processes. As a consequence, Fenton reactions have received great attention in the treatment of domestic and industrial wastewater effluents. Currently, homogeneous and heterogeneous Fenton processes are being investigated intensively and optimized for applications, either alone or in a combination of other processes. This review presents fundamental chemistry involved in various kinds of homogeneous Fenton reactions, which include classical Fenton, electro-Fenton, photo-Fenton, electro-Fenton, sono-electro-Fenton, and solar photoelectron-Fenton. In the homogeneous Fenton reaction process, the molar ratio of iron(II) and hydrogen peroxide, and the $\mathrm{pH}$ usually determine the effectiveness of removing target pollutants and subsequently their mineralization, monitored by a decrease in levels of total organic carbon or chemical oxygen demand. We present catalysts used in heterogeneous Fenton or Fenton-like reactions, such as $\mathrm{H}_{2} \mathrm{O}_{2}-\mathrm{Fe}^{3+}$ (solid)/nano-zero-valent iron/immobilized iron and electro-Fenton-pyrite. Surface properties of heterogeneous catalysts generally control the efficiency to degrade pollutants. Examples of Fenton reactions are demonstrated to degrade and mineralize a wide range of water pollutants in real industrial wastewaters, such as dyes and phenols. Removal of various antibiotics by homogeneous and heterogeneous Fenton reactions is exemplified.
\end{abstract}

Keywords Homogeneous Fenton reaction · Heterogeneous · Advanced oxidation processes · Mechanism · Dyes · Antibiotics

Ajaya Kumar Singh

ajayaksingh_au@yahoo.co.in

* Virender K. Sharma

vsharma@sph.tamhsc.edu

1 Department of Chemistry, Govt. V. Y. T. PG. Autonomous College, Durg, Chhattisgarh 491001, India

2 Department of Energy and Environmental System Engineering, The University of Seoul, 90 Jeonnong-dong, Dongdaemun-gu, Seoul 130-743, Republic of Korea

3 Coll de France, CNRS, INRA, IRD, Europôle Méditerranéen de l'Arbois, CEREGE, Aix-Marseille Univ, 13545 Aix en Provence, France

4 Program for the Environment and Sustainability, Department of Environmental and Occupational Health, School of Public Health, Texas A\&M University, College Station, TX 78843, USA

\section{Introduction}

Water is abundant on earth and is critical to life. Of the total water mass, $97.2 \%$ is present in oceans and seas and $2.1 \%$ exists in glacier. $0.65 \%$ is the fraction of total water mass being utilizable for production of drinking water. This amount contains several lakes' waters which are highly polluted (Bakker 2012; Vörösmarty et al. 2010). Therefore, one of the important issues of this century is to provide clean water to humans (Liu and Yang 2012a, b; Shannon et al. 2008). Based on a recent report of United Nations and World Health Organization (WHO), more than 2 billion humans face some kind of risk to have safe drinking water at home (World Health Organization 2017). Water-related diseases have caused more than 360,000 children die every year under 5 years of age. Ecosystems are also subjected to polluted water-related risks (Schwarzenbach et al. 2006, 2010). 
Water pollutants usually contain diseases-containing agents, e.g., parasitic worms, bacteria, viruses, and protozoa, oxygen-consuming wastes, water soluble inorganics, e.g., toxic metals, nutrients, e.g., phosphates and nitrate, water soluble radioactive compounds and organic pollutants, e.g., plastics, oil, detergents, dyes, aryl chlorides, and pesticides (Kralchevska et al. 2016; Schmeller et al. 2018; Sharma and Sohn 2009; Sousa et al. 2018). In recent years, emerging organic contaminants in water have become major concerns. These include endocrine disruptor chemicals (EDCs) and pharmaceuticals and personal care products (PPCPs) (Cizmas et al. 2015; Hajj-Mohamad et al. 2017; Klatte et al. 2017; Sharma et al. 2016). Pollutants come from industrial, agricultural, and consumer products, which contaminate groundwater and surface water, commonly used as drinking water resources (Blum et al. 2018; Sousa et al. 2018). Water treatments have been greatly investigated for depollution of water for freshwater usage and drinking (Kim et al. 2014; Sharma et al. 2015; Werber et al. 2016).

Various treatment approaches have been applied which include adsorption, biodegradation, coagulation, ionexchange, and oxidation processes (Brillas and MartínezHuitle 2015; Feng et al. 2018; Ghattas et al. 2017; Sharma and Feng 2017). Among these methods, advanced oxidation processes (AOPs) have been researched tremendously for the last two decades (Anumol et al. 2016; Boczkaj and Fernandes 2017; Gassie and Englehardt 2017; Oturan and Aaron 2014). AOPs is based on generation of a powerful oxidizing agent such as hydroxyl radicals $(\mathrm{OH})$ at a significant amount to effectively decontaminate water. Many different kinds of AOPs have been developed to produce in situ
OH radicals (Duan et al. 2018; Liu et al. 2018; Sillanpää et al. 2018). Chemical, sonochemical, photochemical, electrochemical processes have been utilized to form $\mathrm{OH}$ radicals (Brillas and Martínez-Huitle 2015; Cheng et al. 2016; Ganzenko et al. 2017; Garcia-Segura and Brillas 2017; Gligorovski et al. 2015; Sharma 2013; Sirés et al. 2014; Steter et al. 2018; Trellu et al. 2016). The present review pertains to Fenton's reagent, a chemical strategy to efficiently generate in situ $\mathrm{OH}$ radicals. In the literature, many approaches of Fenton reaction have been performed, which include homogeneous and heterogeneous Fenton reactions (Fig. 1). Examples are classical, modifi Fenton reactions (e.g., sono-Fenton, photo-Fenton, electro-Fenton, photo-electroFenton, and ligand assisted Fenton) and solid-solution-based Fenton $\left(\mathrm{H}_{2} \mathrm{O}_{2}\right.$-solid $\mathrm{Fe}^{0}, \mathrm{H}_{2} \mathrm{O}_{2}$-solid $\mathrm{Fe}^{\mathrm{III}}$ (Barbosa et al. 2016; Clarizia et al. 2017; Gligorovski et al. 2015; Mirzaei et al. 2017; Moreira et al. 2016). In the next sections, fundamental chemistry of the Fenton reaction occurring in different systems to generate $\mathrm{OH}$ radicals is presented.

\section{Fenton'sreagent}

A mixture of ferrous ion $\left(\mathrm{Fe}^{2+}\right)$ and hydrogen peroxide $\left(\mathrm{H}_{2} \mathrm{O}_{2}\right)$ is called Fenton's reagent. The chemistry of this reagent started in 1894 when Fenton applied it to degrade tartaric acid (Fenton 1894, 1896). Fenton's reagent involved complex mechanism of reactions, presented in Table 1. Basically, a Fenton process is initiated by the formation of hydroxyl radical ( $\mathrm{OH})$ (reaction F1) (Oturan and Aaron 2014). The reaction F1 takes place in acidic
Fig. 1 Types of Fenton reaction processes used in treating organic pollutants in water

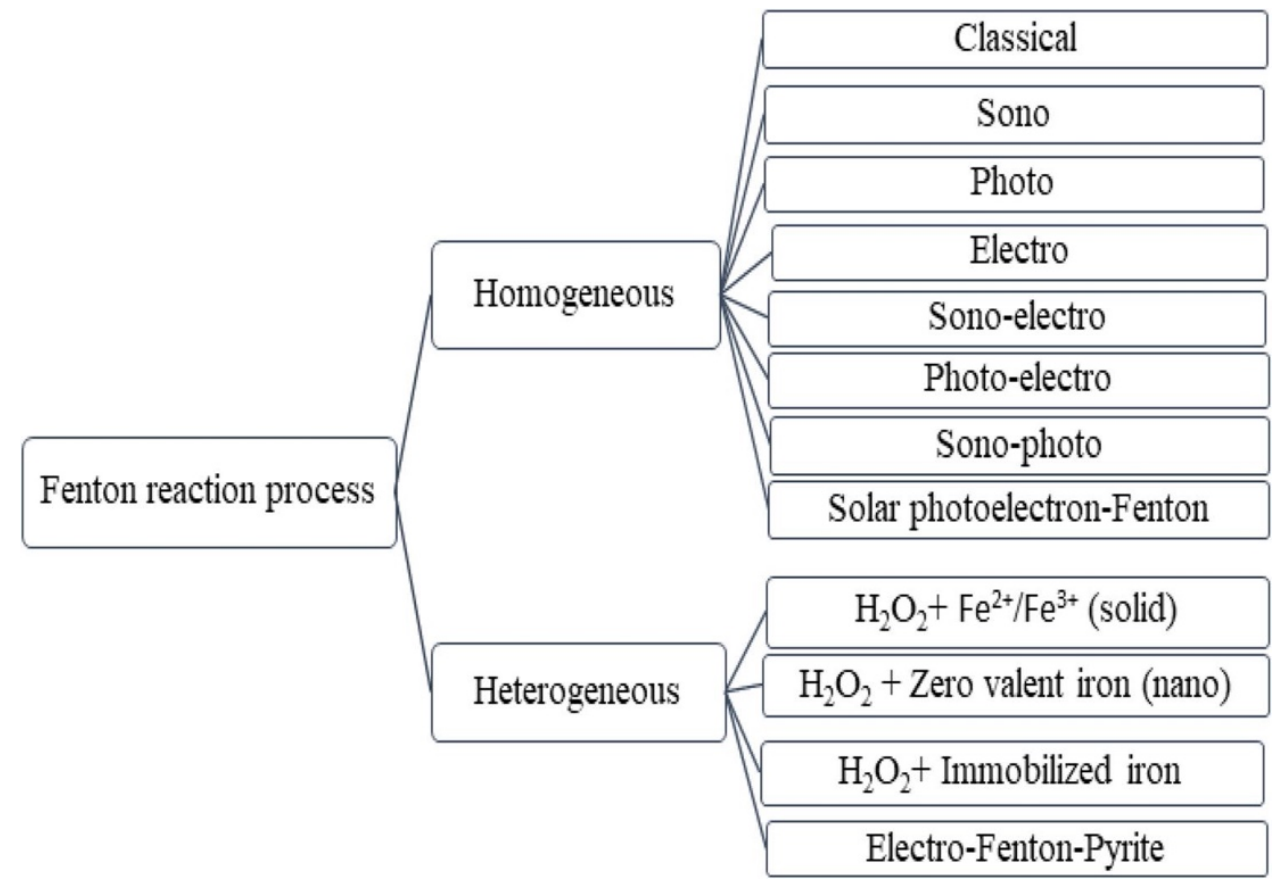


Table 1 Reactions involved in Fenton reaction processes

\begin{tabular}{|c|c|c|}
\hline Fenton's reagent & & \\
\hline $\mathrm{Fe}^{2+}+\mathrm{H}_{2} \mathrm{O}_{2} \rightarrow \mathrm{Fe}^{3+}+\mathrm{OH}+\mathrm{OH}^{-}$ & & \\
\hline $\begin{array}{l}(\mathrm{F} 1) \\
\mathrm{Fe}^{2+}+\mathrm{H}_{2} \mathrm{O}+\mathrm{H}^{+} \rightarrow \mathrm{Fe}^{3+}+\mathrm{H}_{2} \mathrm{O}+\mathrm{OH}\end{array}$ & & (F2) \\
\hline 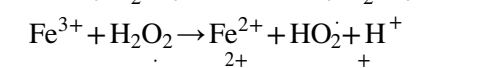 & $\begin{array}{lll}3 & -1 & -1\end{array}$ & (F3) \\
\hline $\begin{array}{l}\mathrm{Fe}_{3+}^{3+}+\mathrm{HO}_{2} \rightarrow \mathrm{Fe}+\mathrm{O}_{2}+\mathrm{H} \\
\mathrm{Fe}^{3+}+\mathrm{O}_{2}^{-} \rightarrow \mathrm{Fe}^{2+}+\mathrm{O}_{2}\end{array}$ & $k_{5}^{4} \equiv 2 \times 187 \mathrm{M}^{-1} \mathrm{~S}^{-1}$ & (F5) \\
\hline $\mathrm{Fe}^{2+}+\mathrm{H}_{2} \mathrm{O}_{2} \rightleftharpoons \mathrm{FeOOH}^{+}+\mathrm{H}^{+}$ & & (F6) \\
\hline $\mathrm{FeOOH}^{+}+\mathrm{H}^{+} \rightleftharpoons \mathrm{Fe}\left(\mathrm{H}_{2} \mathrm{O}_{2}\right)^{2+}$ & & (F7) \\
\hline $\mathrm{Fe}^{2+}+\mathrm{H}_{2} \mathrm{O}_{2} \rightleftharpoons \mathrm{Fe}\left(\mathrm{H}_{2} \mathrm{O}_{2}\right)^{2+}$ & & (F8) \\
\hline $\mathrm{Fe}\left(\mathrm{H}_{2} \mathrm{O}_{2}\right)^{2+} \rightarrow \mathrm{Fe}^{3+}+\mathrm{OH}+\mathrm{OH}^{-}$ & & (F9) \\
\hline $\mathrm{Fe}\left(\mathrm{H}_{2} \mathrm{O}_{2}\right)^{2+} \rightarrow \mathrm{FeO}^{2+}+\mathrm{H}_{2} \mathrm{O}$ & & $(\mathrm{F} 10)$ \\
\hline Electro-Fenton processes & & \\
\hline $\mathrm{Fe}^{3+}+\mathrm{e}^{-} \rightarrow \mathrm{Fe}^{2+}$ & & $(\mathrm{EF} 1)$ \\
\hline $\mathrm{O}_{2}+2 \mathrm{H}^{+}+2 \mathrm{e}^{-} \rightarrow \mathrm{H}_{2} \mathrm{O}_{2}$ & & $(\mathrm{EF} 2)$ \\
\hline $2 \mathrm{H}_{2} \mathrm{O} \rightarrow \mathrm{O}_{2}+4 \mathrm{H}^{+}+4 \mathrm{e}^{-}$ & & (EF3) \\
\hline $\mathrm{Fe} \rightarrow \mathrm{Fe}^{2+}+2 \mathrm{e}^{-}$ & & (EF4) \\
\hline Photo-Fenton processes & & \\
\hline $\mathrm{Fe}^{3+}+h v+\mathrm{H}_{2} \mathrm{O} \rightarrow \mathrm{Fe}^{2+}+\mathrm{OH}+\mathrm{H}^{+}$ & & $(\mathrm{PF} 1)$ \\
\hline $\mathrm{FeOH}^{2+}+h v \rightarrow \mathrm{Fe}^{2+}+\mathrm{OH}$ & & $(\mathrm{PF} 2)$ \\
\hline $\mathrm{Fe}^{3+}-\mathrm{L}+h v \rightarrow \mathrm{Fe}^{2+}+\mathrm{L}^{+}$ & & (PF3) \\
\hline Ultrasound Fenton processes & & \\
\hline $\mathrm{H}+\mathrm{Fe}^{3+} \rightarrow \mathrm{Fe}^{2+}+\mathrm{H}^{+}$ & & (UF1) \\
\hline $\mathrm{H}+\mathrm{O}_{2} \rightarrow \mathrm{HO}_{2}$ & & (UF2) \\
\hline $\begin{array}{l}\mathrm{HO}_{2} \rightleftharpoons \mathrm{O}_{2}^{-}+\mathrm{H}^{+} \\
\mathrm{Fe}^{3+}+\mathrm{O}^{-} \rightarrow \mathrm{Fe}^{2+}+\mathrm{O}_{2}\end{array}$ & & $\begin{array}{l}\text { (UF3) } \\
\text { (UF4) }\end{array}$ \\
\hline $\mathrm{HO}_{2}+\mathrm{O}_{2}^{-2}+\mathrm{H}^{+} \rightarrow \mathrm{H} \mathrm{O}_{2}+\mathrm{O}_{2}$ & & (UF5) \\
\hline $\begin{array}{l}\text { Zero-valent-induced Fenton processes } \\
2 \mathrm{Fe}^{0}+\mathrm{O}_{2}+4 \mathrm{H}^{+} \rightarrow 2 \mathrm{Fe}^{2+}+2 \mathrm{H} \mathrm{O}_{2}\end{array}$ & & $(\mathrm{ZF} 1)$ \\
\hline $\mathrm{Fe}^{2+}++\mathrm{Q}_{2} \rightarrow \mathrm{Fe}^{3+} \rightarrow \mathrm{H}^{3+} \mathrm{O}_{2} \mathrm{O}$ & & (ZE2) \\
\hline $\begin{array}{llll}2 \mathrm{O}_{2} & 2 & 2 & 2 \\
\end{array}$ & & \\
\hline
\end{tabular}

medium, therefore presented by reaction F2. The optimum $\mathrm{pH}$ range for the Fenton process is approximately 2.8-3.0. The catalytic behavior of the redox couple $\mathrm{Fe}^{3+} / \mathrm{Fe}^{2+}$ propagates the Fenton reaction process. For example, only a small amount of $\mathrm{Fe}^{2+}$ is needed because it can be regenerated by the reaction F3, called as Fenton-like reaction. The reaction $\mathrm{F} 3$ produces superoxide radical $\left(\mathrm{HO}^{2}\right)$, which has lower oxidation power $\left(E^{0}=1.65 \mathrm{~V}\right.$ vs. NHE) than hydroxyl radical $\left(E^{0}=2.80 \mathrm{~V}\right.$ vs. NHE). The generation of $\mathrm{Fe}^{2+}$ from the reaction of $\mathrm{Fe}^{3+}$ with superoxide radical (reaction $\mathrm{F} 4$ ) is slower than the reaction $\mathrm{F} 3$. With increase in $\mathrm{pH}$, the protonated superoxide species $\left(\mathrm{HO}_{2}\right)$ converts to the deprotonated species $\left(\mathrm{O}_{2}\right)\left(\mathrm{HO}_{2}=\mathrm{H}+\mathrm{O}_{2} ; \mathrm{p} K_{\mathrm{a}}=4.8\right)($ Czapski and Bielski 1993; Von Sonntag and Schuchmann 1997). The reaction between $\mathrm{Fe}^{3+}$ with $\mathrm{O}_{2}$ (reaction F5) is much faster than reaction F4 (see Table 1) (Bielski and Richter 1977; Gallard and De Laat 2000; Rush and Bielski 1985).

The mechanism of Fenton reaction is still not fully understood. The reaction F1 occurs through inner sphere electron transfer step (Fischbacher et al. 2017; Goldstein and Meyersteion 1999). In the first step, the formation of complex, $\mathrm{FeOOH}^{2+}$, is formed (Gallard et al. 1999) (reaction F6). This complex goes through equilibrium (reaction F7) (Rachmi-

lovich-Calis et al. 2009). Overall reaction of the complex formation is written as reaction F8. A general assumption is that the complex $\mathrm{Fe}\left(\mathrm{H}_{2} \mathrm{O}_{2}\right)^{2+}$ could react through either one-electron or two-electron transfer forming $\mathrm{OH}$ or $\mathrm{Fe}^{\mathrm{IV}}$, respectively (reactions F9 and F10) (Bataineh et al. 2012; Hug and Leupin 2003; Katsoyiannis et al. 2008; Von Sonn$\operatorname{tag}$ 2008).

Advantages of Fenton's reagent are that it is simple and easy to apply without any requirement of energy input (Bautista et al. 2008). However, Fenton's reagent has some drawbacks which include risk of storage of hydrogen peroxide, adjustment of $\mathrm{pH}$ to acidic range, and buildup of iron sludge (Oturan and Aaron 2014). Optimizing the dosages of reactants may minimize disadvantages of Fenton's reagent. Use of iron oxides, iron-modified clays, ion-exchange resins, iron-exchange Nafion membranes, and zeolites, and alumina may reduce the generation of sludge (Bautista et al. 2008; Lucas et al. 2007; Pignatello et al. 2006; Zhang et al. 2011a, b). Fenton's reagent has been applied to degrade and subsequent destruction and mineralization of numerous organic pollutants (Aljuboury et al. 2017; Annabi et al. 2016; Descorme 2017; Ganzenko et al. 2017; Li et al. 2016; Tayo et al. 2018; Usman et al. 2016). Examples include treatment of dyes, phenols, chlorophenols, chlorobenzenes, and antibiotics. Details are described in later sections of the review.

\section{Electro-Fenton process}

In the electro-Fenton process, $\mathrm{Fe}^{2+}$ and $\mathrm{H}_{2} \mathrm{O}_{2}$ are produced simultaneously by cathodic reduction of $\mathrm{Fe}^{3+}$ and $\mathrm{O}_{2}$, respectively (reactions EF1 and EF2, Table 1) (Barhoumi et al. 2015, 2016; He and Zhou 2017; Lin et al. 2017a; Mousset et al. 2018; Mousset et al. 2016, 2018; Nidheesh et al. 2018; Sirés et al. 2014; Wang et al. 2016). A small amount of salt of $\mathrm{Fe}^{2+}$ (e.g., ferrous sulfate) is initially added, which can react with electrochemically produced $\mathrm{H}_{2} \mathrm{O}_{2}$ to produce $\mathrm{Fe}^{3+}$. Reaction $\mathrm{EF} 1$ is critical to carry out recycling of $\mathrm{Fe}^{3+}$ to $\mathrm{Fe}^{2+}$. Glassy carbon and graphite are rarely used as cathodes in electro-Fenton process. The most often used electro-Fenton's cathodes are carbon felt (Oturan et al. 2008) and GDE (gas diffusion electrode) cathodes (Brillas et al. 2009; Oturan and Aaron 2014). These are 3D cathodes. A platinum electrode is applied to perform the anodic reaction in the laboratory setup (reaction EF3, Table 1). In the peroxycoagulation, fered Fenton, anodic Fenton treatment or electrochemical peroxidation, $\mathrm{Fe}^{2+}$ can also be produced from the oxidation of sacrifi anode of iron (reaction EF4, Table 1) (Brillas et al. 2009). A few reviews on the 
elimination of pollutants including micro-pollutants in water using electro-Fenton process have been published (Annabi et al. 2016; Brillas et al. 2009; Isarain-Chávez et al. 2011; Lin et al. 2017b; Steter et al. 2018; Wang et al. 2015; Özcan et al. 2016). A progress is being made to enhance the efficiency of electro-Fenton process. A research on sonoelectro-Fenton and photo-Fenton processes has also been performed in order to improve efficiency and practicality of electro-Fenton methods (Bocos et al. 2016; Espinoza et al. 2016; Gozzi et al. 2017; Kalishwaralal et al. 2016; Oturan et al. 2008; Pliego et al. 2015; Uribe et al. 2015; Vidal et al. 2018). Electro-Fenton process is also being combined with biological methods to mineralize organic pollutants in water (Annabi et al. 2016; Ganzenko et al. 2017). The coupling of electro-Fenton with biological degradation is a new and interesting tool. The coupled process is called bioelectroFenton process (Olvera-Vargas et al. 2016a, b).

\section{Photo-Fenton process}

In the Photo-Fenton process, the ultraviolet (UV) light can assist the reduction of $\mathrm{Fe}^{3+}$ to $\mathrm{Fe}^{2+}$ (reaction PF1, Table 1) to react with $\mathrm{H}_{2} \mathrm{O}_{2}$ to generate $\mathrm{OH}$ through the reaction F1 (Table 1). Effi of reaction is greatest at $\mathrm{pH} 3.0$ because $\mathrm{Fe}^{3+}$ ions generally exist as $\mathrm{Fe}(\mathrm{OH})^{2+}$ under this condition. The photo-Fenton process has possibility of using many several UV regions as light energy source, namely UVA $(\lambda=315-400 \mathrm{~nm})$, UVB $(\lambda=285-315 \mathrm{~nm})$, and UVC $(\lambda<285 \mathrm{~nm})$; therefore, the yield of $\mathrm{OH}$ varies with intensity of light. The $\mathrm{Fe}(\mathrm{OH})^{2+}$ has absorbance maximum only in the UVB region. Production of $\mathrm{OH}$ through the UV photolysis of $\mathrm{Fe}(\mathrm{OH})^{2+}$ is low (quantum yield of reaction PF2 is 0.2) (Pignatello et al. 2006; Zepp et al. 1992). Furthermore, solar light has fraction of light in the UVB region; only limited solar light irradiation can be absorbed. At the neutral $\mathrm{pH}$, $\mathrm{Fe}^{3+}$ precipitated out and efficiency of photo-Fenton process is quite low. This drawback of the photo-Fenton process can be minimized by adding ligands (L) such as polycarboxylates and polyaminocarboxylates (e.g., oxalate, citrate, ethylenediaminetetraacetatic acid, and ethylenediamine $N, N^{\prime}-$ disuccinic acid) (Faust and Hoigné 1990; Faust and Zepp 1993; Li et al. 2012; Weller et al. 2013a, b). These ligands form stable complexes with $\mathrm{Fe}^{3+}$, which upon UV and visible light irradiation reduce $\mathrm{Fe}^{3+}$ to $\mathrm{Fe}^{2+}$ via ligand-tometal-charge transfer (LMCT) step (reaction PF3, Table 1). Quantum yields of these $\mathrm{Fe}^{3+}$-L complexes are higher than quantum yield of $\mathrm{Fe}(\mathrm{OH})^{2+}$. The use of organic ligand complexes of $\mathrm{Fe}^{3+}$ is advantageous. However, the ligands are attacked by $\mathrm{OH}$ produced in the process reducing the efficiency. Photo-Fenton process has shown their effectiveness in removing a wide range of contaminants, which include polychlorinated biphenyls, pesticides, and pharmaceuticals
(Clarizia et al. 2017; Gligorovski et al. 2015; Matafonova and Batoev 2018; Serpone et al. 2017).

\section{Ultrasound Fentonprocess}

In the ultrasound Fenton process, a high-frequency is applied to split water into $\mathrm{OH}$ and $\mathrm{H}$ radicals (Eren 2012; Ma 2012; Salimi et al. 2017; Özdemir et al. 2011). Sonolysis of solution containing $\mathrm{Fe}^{3+}$ ions results in a series of reactions (reactions UF1-UF5, Table 1) to generate both $\mathrm{Fe}^{2+}$ and $\mathrm{H}_{2} \mathrm{O}_{2}$ for Fenton reaction (Gligorovski et al. 2015). Basically, sonochemistry and Fenton reaction generate $\mathrm{OH}$ to carry out transformation of organic pollutants in water (Chakma and Moholkar 2014, 2015). Several investigations have been explored applications of ultrasound Fenton processes to degrade a number of contaminants in water (Durán et al. 2013; Feng et al. 2013).

\section{Heterogeneous Fenton reaction}

A heterogeneous Fenton-like process has been investigated by many researchers because of advantages over homogeneous Fenton reactions (Cai et al. 2016; Diao et al. 2017; García-Rodríguez et al. 2017; Li et al. 2018; Lyu and Hu 2017; Velichkova et al. 2017). One of the main advantages of heterogeneous Fenton reactions is its feasibility over a wide $\mathrm{pH}$ rage to degrade pollutants in water. If the source of iron is magnetic like magnetite $\left(\mathrm{Fe}_{3} \mathrm{O}_{4}\right)$, a magnetic separation can be applied (Morales-Pérez et al. 2016b). A focus of the research in the heterogeneous Fenton process is to increase the catalytic activity of solid iron sources without leaching of iron to aqueous environment. Efforts have been made to characterize catalysts for their pore size, density, and porosity in order to achieve better catalytic efficiency of heterogeneous Fenton-like reactions. A number of catalysts have been applied to carry out heterogeneous Fenton reactions (Costa et al. 2008; Diao et al. 2017, 2018; García-Rodríguez et al. 2017; Mirzaei et al. 2017; Morales-Pérez et al. 2016a; Nidheesh et al. 2017; Oturan et al. 2018; Ouiriemmi et al. 2017; Pi et al. 2018).

The $\mathrm{OH}$ radicals may also be produced in a reductive environment (Cao et al. 2013; Le et al. 2011; Vilardi et al. 2018). It has been shown that an addition of zero-valent iron $\left(\mathrm{ZVI}, \mathrm{Fe}^{0}\right)$ to an aerated water solution yields the precursors reactants $\left(\mathrm{Fe}^{2+}\right.$ and $\left.\mathrm{H}_{2} \mathrm{O}_{2}\right)$ through a sequence of reactions (ZF1-ZF3, Table 1) (Kang et al. 2017). In the presence of organic compounds and ZVI, many additional reactions also occur. This may be the reason of limited use of ZVI in Fenton reaction processes (Shimizu et al. 2012).

Iron oxides minerals like pyrite $\left(\mathrm{FeS}_{2}\right)$, hematite $\left(\mathrm{Fe}_{2} \mathrm{O}_{3}\right)$, goethite $(\alpha-\mathrm{FeOOH})$, and lepidocrocite $(\gamma-\mathrm{FeOOH})$ have 
been studied in the heterogeneous Fenton-like reactions. Numerous support materials in heterogeneous Fenton processes have also been used. These include activated carbon, zeolites, clays, silicas, layered materials, and graphene oxide (Espinosa et al. 2016). More recently, a focus is on metal nanoparticles (e.g., $\mathrm{Ag}, \mathrm{Cu}$, and $\mathrm{Au}$ ) to modify surfaces to obtain more eff eness of the heterogeneous Fenton reaction processes (Dhakshinamoorthy et al. 2012; Espinosa et al. 2018). Details of a range of catalysts in Fenton reactions are given in reviews on the subject of degradation of several kinds of pollutants in water.

In following section of the review, examples are given to demonstrate applications of homogeneous and heterogeneous Fenton reaction processes.

\section{Treatment of industrial wastewater: homogeneous Fenton reaction process}

Effl released from various industries contain pollutants at high levels, which could be a threat to human life. Removal of these pollutants by biological and eco-friendly methods is not successful as industrial waste contains mainly organic loads comprising of high COD and BOD. In developing countries, $85-90 \%$ of the wastewater is discharged directly into surface water bodies without proper treatment, and thus, the pollutants present in the discharged directly to the environment (Shannon et al. 2008). In India, nearly 6.2 million $\mathrm{m}^{3}$ of industrial wastewater is generated every day, and only $60 \%$ of it is being treated (Kaur et al. 2012).

Chemical method is considered as a convenient strategy for removing these pollutants. Among various chemical processes, advanced oxidation process has found to be appropriate approach to minimize contamination from industrial effl Table 2 presents examples of Fenton treatment of industrial waste generated by various industries of the world. A wide range of chemicals have been found in industrial wastewater, which include pesticides and pharmaceuticals. Generally, industrial wastewater contains high values of chemical oxygen demand (COD), biological demand (BOD), dissolved organic carbon (DOC), and total organic carbon. Fenton's reagent was applied at various molar ratios of $\mathrm{Fe}$ (II) and $\mathrm{H}_{2} \mathrm{O}_{2}$ (Table 2). Pollutants of industrial wastewater could be degraded almost completely. Moreover, results showed a significant decrease in values of COD, BOD, and DOC after the treatment with Fenton's reagent. For example, at a molar ratio 10 for $0.2 \mathrm{mM} \mathrm{Fe}^{3+}$, TOC removal was $90 \%$ in real industrial water (Bouafi gui et al. 2010). This suggests that mineralization of organic pollutants (e.g., maleic acid anhydride, pesticides, 2-ethylhexyl alcohol, urea formaldehyde resin adhesive, $\alpha, \beta, \gamma-\mathrm{HCH}$, DDT, DMDT, fenitrothion, chlorfenvinphos) could be achieved using the
Fenton's reagent. Values of DOC decreased signifi y by the treatment with the Fenton's reagent. Components of petroleum waste like ethylene glycol, 1,4-dioxane, lower $[\mathrm{Fe}(\mathrm{II})] /\left[\mathrm{H}_{2} \mathrm{O}_{2}\right]$ value could also be degraded at molar ratio of $\mathrm{Fe}$ (II) to $\mathrm{H}_{2} \mathrm{O}_{2}$ as 0.02 .

\section{Degradation of pollutants: homogeneous Fenton reaction processes}

Degradation of representative phenols and pesticides by homogeneous Fenton reaction is given in Table 3. Phenol and dichlorvos were found to be successfully removed by homogeneous Fenton reaction in the acidic $\mathrm{pH}$ range (Table 3). Only $80 \%$ removal of 2,4-dichlorophenol was seen. Transformation of bisphenol A formed various intermediates, suggested not signifi ant mineralization of parent molecule under studied conditions. Homogeneous Fenton reaction in combination with ultrasound was highly eff es in degrading diff ent kind of pesticides. Removal of the pesticides was almost complete (Table 3).

\section{Degradation of dyes: heterogeneous Fenton reaction processes}

Nowadays, dyes are frequently used for several purposes such as dyeing clothes, leather, furniture, even in our regular life in food, cosmetics, and medicine, etc. Dyes are not easily degraded because some of them are non-biodegradable and have long-term adverse eff Dyes have shown hazard eff on environment after entering into the ecosystem (Huang et al. 2009). Dyes can be degraded by various methods, including biomass degradation (Hsu et al. 2012; Prachi and Anushree 2009), photocatalytic degradation (Gu et al. 2014), combined treatment (Jafari et al. 2012). Among various advanced oxidation methods, Fenton reaction systems are reliable methods to transform dyes into many smaller fragments, i.e., water, carbon dioxide. Examples of degrading dyes by Heterogeneous Fenton reactions systems are summarized in Table 4. Catalysts used in the systems were iron- and carbon-based materials. Combinations of iron and carbon materials were also utilized to degrade dyes. Signifi y, most of the studies shown in Table 4 under diff ent conditions and catalysts could achieve the complete degradation of dyes. However, time of the complete degradation varied with experimental conditions. Nanocomposite materials in heterogeneous Fenton reaction systems seem to take less time to obtain the complete degradation compared to other catalysts. 
Table 2 Treatment of real industrial wastewater by homogeneous Fenton reaction process. COD—chemical oxygen demand, BOD—biological oxygen demand, DOC - dissolved organic carbon, TOC—-total oxygen carbon

\begin{tabular}{llll}
\hline No. Region & Pollutants & $\begin{array}{l}\text { Reaction conditions and com- } \\
\text { ments }\end{array}$ & References
\end{tabular}

1. Tambla Tributary (River

Damodar), India

2. Chemical factories, Southern Poland

3. Pesticide-containing wastewater, Southern Poland

4. Pharmaceutical waste water, Turkey

5. Laboratory mixed waste chemical of 17-month period, Brazil

6. Fish canning waste water, Portugal

7. Chemical plant that produce acrylic sheets, Mexico

8. Real industrial biorecalcitrant wastewater, Spain

9. El-Nasr pharmaceutical and Chemical Company, South-East of Cairo

10. Tannery wastewater, Brazil

11. Petrochemical effluent, India

12. Pesticides wastewater from Nubaria, Egypt ariety of pharmaceutical chemicals

Industrial waste

$[\mathrm{Fe}(\mathrm{II})]=6 \mathrm{~g} / \mathrm{L}$ and

$\left[\mathrm{H}_{2} \mathrm{O}_{2}\right]=220 \mathrm{~g} / \mathrm{L}$

COD removal $=\sim 95 \%$

Maleic acid anhydride, pesticides, $[\mathrm{Fe}(\mathrm{II})] /\left[\mathrm{H}_{2} \mathrm{O}_{2}\right]=0.33$, (maleic 2-ethylhexyl alcohol, urea formaldehyde resin adhesive

$\alpha, \beta, \gamma-\mathrm{HCH}, \mathrm{DDT}$, DMDT, fenitrothion, chlorfenvinphos

Different laboratory chemicals waste

Organic matter, salts, oil and grease

Methyl methacrylate

5-Amino-6-methyl-2-benzimidazolone

Pharmaceutical company discharges both industrial and municipal wastewater

Containing both organic and inorganic pollutants

Ethylene glycol, 1,4-dioxane

Chlorpyrifos, lambda-cyhalothrin, diazinon acid anhydride), $\mathrm{pH}=3.0, \mathrm{COD}$

removal $=\sim 88 \%$

$[\mathrm{Fe}(\mathrm{II})] /\left[\mathrm{H}_{2} \mathrm{O}_{2}\right]=0.50$, (2-ethylhexyl alcohol), $\mathrm{pH}=3.5, \mathrm{COD}$ removal $=86.3 \%$

$[\mathrm{Fe}(\mathrm{II})] /\left[\mathrm{H}_{2} \mathrm{O}_{2}\right]=0.33$, (urea formaldehyde resin adhesive),

$\mathrm{pH}=3.5$, COD removal $=88.8 \%$

$[\mathrm{Fe}(\mathrm{II})] /\left[\mathrm{H}_{2} \mathrm{O}_{2}\right]=0.33$, (pesticide containing water), $\mathrm{pH}=3.2$ COD removal $=71.7 \%$

All pesticides degraded completely

$\left[\mathrm{H}_{2} \mathrm{O}_{2}\right]=5 \mathrm{~g} / \mathrm{L}$ and $[\mathrm{Fe}(\mathrm{II})] /$ $\left[\mathrm{H}_{2} \mathrm{O}_{2}\right]=0.33-0.50$ Fenton oxidation followed by sequencing batch reactor, COD removal $=\sim 99 \%$

$[\mathrm{Fe}(\mathrm{II})] /\left[\mathrm{H}_{2} \mathrm{O}_{2}\right]=0.22, \mathrm{COD}$ removal $=90 \%$

Before treatment:

$\mathrm{DOC}=50 \mathrm{mg} / \mathrm{L}$,

$\mathrm{COD}=220 \mathrm{mg} / \mathrm{L}$,

$\mathrm{BOD}_{5}=0.8 \mathrm{mg} / \mathrm{L}$

(Biological pretreatment of fish canning waste water, followed by treatment with Fenton's reagent)

After treatment: $\mathrm{DOC}=20 \mathrm{mg} / \mathrm{L}$, $\mathrm{COD}=90 \mathrm{mg} / \mathrm{L}$

Maximum removal efficiencies (Fenton adsorption treatment): $96 \%$ color, $58 \%$ TOC, and $60 \%$ COD

$[\mathrm{Fe}(\mathrm{II})] /\left[\mathrm{H}_{2} \mathrm{O}_{2}\right]=0.0032$

Removal: $67 \%$ color, $42 \%$ COD, and $41 \%$ TOC

Before treatment: COD (4100$13,023 \mathrm{mg} / \mathrm{L})$, TSS

$(20-330 \mathrm{mg} / \mathrm{L})$, and oil grease (17.4-600 mg/L)

Treatment: $[\mathrm{Fe}(\mathrm{II})] /\left[\mathrm{H}_{2} \mathrm{O}_{2}\right]=0.02$ and $\mathrm{COD} /\left[\mathrm{H}_{2} \mathrm{O}_{2}\right]=1: 2.2$

Removal was almost complete

$\mathrm{H}_{2} \mathrm{O}_{2} / \mathrm{UV}$ at $\mathrm{pH} 3$ and Fenton at pH 3.5; efficiently remove TOC to low level

$[\mathrm{Fe}(\mathrm{II})] /\left[\mathrm{H}_{2} \mathrm{O}_{2}\right]=0.02 ; \mathrm{pH} 3.0$

COD removal $=97.5 \%$

COD removal (photo-Fenton process $)=90.7 \%$

COD removal $\left(\mathrm{TiO}_{2}\right.$ photocatalytic reaction $=79.6 \%$
Mandal et al. (2010)

Barbusinski (2005)

Barbusinski and Filipek (2001)

Tekin et al. (2006)

Benatti and Tavares (2012)

Cristovao et al. (2014)

Sanchez et al. (2014)

Sarria et al. (2001)

Badawy et al. (2009)

Schrank et al. (2005)

Ghosh et al. (2011)

Alalm et al. (2015) 
Table 2 (continued)

\begin{tabular}{|c|c|c|c|c|}
\hline No. & Region & Pollutants & $\begin{array}{l}\text { Reaction conditions and com- } \\
\text { ments }\end{array}$ & References \\
\hline 13. & $\begin{array}{l}\text { Wastewater obtained from civilian } \\
\text { explosive industry, South-west, } \\
\text { China }\end{array}$ & Dinitrodiazophenol (DDNP) & $\begin{array}{l}\text { Treatment: combined } \mathrm{Fe}^{0} / \text { air and } \\
\text { Fenton process } \\
\text { COD removal }=78 \% \\
\text { Chromaticity removal }=98 \% \\
\text { chromaticity }\end{array}$ & Yuan et al. (2016) \\
\hline 14. & $\begin{array}{l}\text { Beverage industrial effluent, } \\
\text { Spain }\end{array}$ & Different complex compound & $\begin{array}{l}\text { Photo-Fenton process): } 53 \% \\
\text { mineralization }(2 \mathrm{~h}) \\
\text { Photo-Fenton/persulfate: } 76 \% \\
\text { mineralization }(4 \mathrm{~h})\end{array}$ & Exposito et al. (2016) \\
\hline 15 . & . Winery wastewater, Cyprus & Polyphenols, tannins, and lignins & 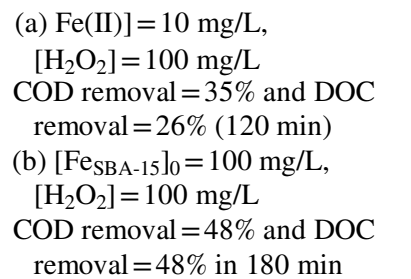 & Loannou et al. (2013) \\
\hline
\end{tabular}

Table 3 Examples of degradation of phenols, pesticides, and surfactants in water by the homogeneous Fenton reaction

\begin{tabular}{|c|c|c|c|c|}
\hline No. & Pollutants & Reaction conditions & Results and comments & References \\
\hline 1. & Phenol & $\begin{array}{l}{\left[\mathrm{H}_{2} \mathrm{O}_{2}\right]=300-600 \mathrm{mg} / \mathrm{L}} \\
\mathrm{Fe}(\mathrm{II})=10 \mathrm{mg} / \mathrm{L} ; \mathrm{pH}=3-3.5, \text { room } \\
\text { temperature; reaction time }=6 \mathrm{~h}\end{array}$ & $\begin{array}{l}\text { Degradation }=100 \% \\
\text { Mineralization }=60 \%\end{array}$ & Yalfani et al. (2009) \\
\hline 2. & 2,4-dichlorophenol & $\begin{array}{l}{[2,4-\text { Dichlorophenol }]=200 \mathrm{mg} / \mathrm{L}} \\
{\left[\mathrm{H}_{2} \mathrm{O}_{2}\right]=300-580 \mathrm{mg} / \mathrm{L}} \\
{[\mathrm{Fe}(\mathrm{II})]=10-20 \mathrm{mg} / \mathrm{L} ; \mathrm{pH}=2.5-7.0}\end{array}$ & Removal $=\sim 70 \%$ within $2 \mathrm{~h}$ & Ranjit et al. (2008) \\
\hline 3. & Bisphenol A (BPA) & $\begin{array}{l}{[\mathrm{BPA}]=10 \mu \mathrm{g} / \mathrm{L}, \mathrm{pH}=3,[\mathrm{Fe}(\mathrm{II})] /} \\
{\left[\mathrm{H}_{2} \mathrm{O}_{2}\right]=10}\end{array}$ & $\begin{array}{l}\text { Degradation resulted in various } \\
\text { intermediates, benzenediols, } \\
\text { monohydroxylated BPA with } \\
\text { molecular weight ranged from } \\
94 \text { to } 500 \mathrm{Da}\end{array}$ & Poerscchmann et al. (2010) \\
\hline 4. & $\begin{array}{l}\text { Dichlorvos or 2,2-dichlorovinyl } \\
\text { dimethyl phosphate }\end{array}$ & $\begin{array}{l}{\left[\mathrm{H}_{2} \mathrm{O}_{2}\right]=15 \mathrm{mg} / \mathrm{L},[\mathrm{Fe}(\mathrm{II})] /\left[\mathrm{H}_{2} \mathrm{O}_{2}\right]=3.0,} \\
\mathrm{pH}=3.0, \text { room temperature; ultrasonic } \\
\text { probe frequency }=20 \mathrm{kHz}\end{array}$ & Degradation $=100 \%$ & Golash and Gogate (2012) \\
\hline 5. & $\begin{array}{l}\text { Methyl parathion (Phosphate } \\
\text { pesticides) }\end{array}$ & $\begin{aligned} & {[\text { Methyl parathion }]=20 \mathrm{mg} / \mathrm{L} } \\
& {\left[\mathrm{H}_{2} \mathrm{O}_{2}\right] }=200 \mathrm{mg} / \mathrm{L} ;[\mathrm{Fe}(\mathrm{II})] / \\
& {\left[\mathrm{H}_{2} \mathrm{O}_{2}\right] }=3.0\end{aligned}$ & $\begin{array}{l}\text { Degradation (ultrasonic horn } \\
\text { process) }=\sim 98.5 \% \text { TOC } \\
\text { removal }=73.7 \% \\
\text { Degradation (ultrasonic bath } \\
\text { process) }=\sim 96.5 \% \\
\text { TOC removal (ultrasonic bath } \\
\text { process) }=75 \%\end{array}$ & Shriwas and Gogate (2011) \\
\hline 6. & $\begin{array}{l}\text { Carbofuran (carbamate pesti- } \\
\text { cides) }\end{array}$ & $\begin{array}{l}{[\text { Carbofuran }]=20 \mathrm{mg} / \mathrm{L},} \\
{\left[\mathrm{H}_{2} \mathrm{O}_{2}\right]=100 \mathrm{mg} / \mathrm{L},[\mathrm{Fe}(\mathrm{II})]=20 \mathrm{mg} / \mathrm{L}}\end{array}$ & $\begin{array}{l}\text { Degradation }=\sim 99 \% \\
\text { Mineralization }=46 \% \text { after } \\
30 \text { min }\end{array}$ & Ma et al. (2010) \\
\hline
\end{tabular}

\section{Treatment of antibiotics}

Human and veterinary antibiotics are considered to be of prime importance as emerging micro-pollutants due to their high consumption rate. These micro-pollutants are being generated through household, industry, hospital service, poultry, livestock, and aquatic activity which get deposited and leached into the environment. The fate of antibiotics after their purposive use is not being monitored. Most of the antibiotics are not fully eliminated from the body, and some of them may remain unchanged in the environment (Hirsch et al. 1999; Brown et al. 2006). Thus, in order to bring awareness among the people, world antibiotics week is being organized since November 2015, with the theme "antibiotics: Handle with care." Antibiotics 
Table 4 Examples of degradation of dyes by heterogeneous Fenton reaction processes

\begin{tabular}{|c|c|c|c|c|c|}
\hline No. & Dyes & Catalyst & Reaction conditions & Results & References \\
\hline 1. & Acid black 1 & $\begin{array}{l}\text { Pillared laponite clay- } \\
\text { based } \mathrm{Fe}\end{array}$ & $\begin{array}{l}{[\text { Dye }]=0.2 \mathrm{mM} ;} \\
{[\text { Catalyst }]=1 \mathrm{~g} / \mathrm{L},} \\
{\left[\mathrm{H}_{2} \mathrm{O}_{2}\right]=6.4 \mathrm{mM}, \text { Light }} \\
\text { intensity }=8 \mathrm{~W} \text { UVC }\end{array}$ & Removal $=100 \%$ in $60 \mathrm{~min}$ & Sum et al. (2004) \\
\hline 2. & Acid blue 185 & $\begin{array}{l}\text { Natural and ball-milled } \\
\text { magnetite nanostructures }\end{array}$ & $\begin{array}{l}{[\text { Dye }]=20-120 \mathrm{mg} / \mathrm{L}} \\
{[\text { Catalyst }]=1.5 \mathrm{~g} / \mathrm{L}} \\
\mathrm{pH}=3.0\end{array}$ & $\begin{array}{l}\text { Removal }=80-99 \% \\
\text { 6-h ball-milled mag- } \\
\text { netite showed highest } \\
\text { efficiency }\end{array}$ & Acisli et al. (2017) \\
\hline 3. & Acid blue 74 & Fe-ZSM5 zeolite & $\begin{array}{c}{[\text { Dye }]=8.56 \times 10^{-5} \mathrm{~mol} / \mathrm{L},} \\
{\left[\mathrm{H}_{2} \mathrm{O}_{2}\right]=21.2 \mathrm{mmol} / \mathrm{L},} \\
{[\text { Catalyst }]=0.5 \mathrm{~g} / \mathrm{L}, \mathrm{pH}} \\
5,0, \mathrm{UV} \text { irradiation }\end{array}$ & $\begin{array}{l}\text { Removal }=100 \% \text { in } \\
120 \text { min }\end{array}$ & Kasiri et al. (2008) \\
\hline 4. & Acid blue 92 & $\begin{array}{l}\text { Natural martite prepared } \\
\text { by ball milling }\end{array}$ & $\begin{array}{l}{[\text { Dye }]=10 \mathrm{mg} / \mathrm{L}} \\
{[\text { Catalyst }]=2.5 \mathrm{~g} / \mathrm{L}} \\
\mathrm{pH}=7.0,2.5 \mathrm{~g} / \mathrm{L} \\
\text { Ultrasonic power }=150 \mathrm{~W}\end{array}$ & $\begin{array}{l}\text { Removal }=100 \% \text { in } 30 \mathrm{~min} \\
\text { achieved }\end{array}$ & Dindarsafa et al. (2017) \\
\hline 5. & Acid orange 7 & $\begin{array}{l}\text { Graphene oxide-iron oxide } \\
\text { nanocomposites }\end{array}$ & $\begin{array}{l}{[\text { Dye }]=35 \mathrm{mg} / \mathrm{L}} \\
{[\text { Catalyst }]=0.2 \mathrm{~g} / \mathrm{L}} \\
{\left[\mathrm{H}_{2} \mathrm{O}_{2}\right]=2 \mathrm{mM}} \\
\mathrm{pH}=3.0\end{array}$ & $\begin{array}{l}\text { Removal }=80 \% \text { in } 20 \mathrm{~min} \\
\text { Removal }=98 \% \text { in } 180 \mathrm{~min}\end{array}$ & Zubir et al. (2014a, b) \\
\hline 6. & Acid orange 7 & $\begin{array}{l}\text { Graphene oxide-iron oxide } \\
\text { nanocomposites }\end{array}$ & $\begin{array}{l}{[\text { Dye }]=0.1 \mathrm{mM}} \\
{[\text { Catalyst }]=0.2 \mathrm{~g} / \mathrm{L}} \\
{\left[\mathrm{H}_{2} \mathrm{O}_{2}\right]=22 \mathrm{mM}} \\
\mathrm{pH}=3.0\end{array}$ & Removal $=96 \%$ on $90 \mathrm{~min}$ & Zubir et al. (2014a, b) \\
\hline 7. & Anthraquinone dye & $\begin{array}{l}\text { Pyrite nanorods synthesize } \\
\text { by oxygen and nitrogen } \\
\text { nonthermal plasma }\end{array}$ & $\begin{array}{l}{[\text { Dye }]=20 \mathrm{mg} / \mathrm{L}} \\
{[\text { Catalyst }]=0.6 \mathrm{~g} / \mathrm{L}} \\
\mathrm{pH}=5.0\end{array}$ & Removal $=100 \%$ in $40 \mathrm{~min}$ & Khataee et al. (2016) \\
\hline 8. & Brilliant orange $\mathrm{X}-\mathrm{GN}$ & $\begin{array}{l}\text { Iron-pillared montmoril- } \\
\text { lonitic via pillaring }\end{array}$ & $\begin{array}{l}\text { Ultrasonic power }=300 \mathrm{~W} \\
{[\text { Dye }]=100 \mathrm{mg} / \mathrm{L}} \\
{[\text { Catalyst }]=0.6 \mathrm{~g} / \mathrm{L}} \\
\text { catalyst, } \\
{\left[\mathrm{H}_{2} \mathrm{O}_{2}\right]=4.9 \mathrm{mmol} / \mathrm{L}} \\
\mathrm{H}_{2} \mathrm{O}_{2} \text { are } \mathrm{pH}=3.0\end{array}$ & $\begin{array}{l}\text { Removal }=98.6 \% \text { in } \\
140 \text { min } \\
\text { Under UV light } \\
\text { Removal }=80 \% \text { in } 140 \mathrm{~min} \\
\text { under visible light }\end{array}$ & Chen et al. (2009) \\
\hline 9. & $\begin{array}{l}\text { 1-Diazo-2-naphthol-4-sul- } \\
\text { fonic acid dye }\end{array}$ & Mesoporous carbon-Fe & $\begin{array}{l}{[\text { Dye }]=250 \mathrm{mg} / \mathrm{L}} \\
{[\text { Catalyst }]=0.5 \mathrm{~g} / \mathrm{L}} \\
\mathrm{pH}=5.0\end{array}$ & Removal $=94 \%$ in $120 \mathrm{~min}$ & Gu et al. (2013) \\
\hline 10. & Methylene blue & $\mathrm{Fe}_{3} \mathrm{O}_{4}-\mathrm{MWCNT}$ & $\begin{array}{l}{[\text { Dye }]=10 \mathrm{mg} / \mathrm{L}} \\
{[\text { Catalyst }]=0.3 \mathrm{~g} / \mathrm{L}} \\
{\left[\mathrm{H}_{2} \mathrm{O}_{2}\right]=0.4 \mathrm{M}} \\
\text { pH } 5.5\end{array}$ & Removal $=97 \%$ in $720 \mathrm{~min}$ & Wang et al. (2014) \\
\hline 11. & Orange II & Fe/ZSM-5 zeolite & $\begin{array}{l}{[\text { Dye }]=0.1 \mathrm{mM}} \\
{[\text { Catalyst }]=200 \mathrm{mg} / \mathrm{L}} \\
{\left[\mathrm{H}_{2} \mathrm{O}_{2}\right]=6 \mathrm{mM}} \\
\text { Temperature }=53{ }^{\circ} \mathrm{C} \\
\text { pH=5.2 }\end{array}$ & $\begin{array}{l}\text { Removal }=100 \% \text { in } \\
240 \text { min }\end{array}$ & Duarte et al. (2009) \\
\hline 12. & Orange II & $\begin{array}{l}\text { Transition metal on carbon } \\
\text { aerogels }\end{array}$ & $\begin{array}{l}{[\text { Dye }]=0.1 \mathrm{mM}} \\
{[\text { Catalyst }]=0.2 \mathrm{~g} / \mathrm{L}} \\
{\left[\mathrm{H}_{2} \mathrm{O}_{2}\right]=6.0 \mathrm{mM}} \\
\mathrm{pH}=3.0\end{array}$ & $\begin{array}{l}\text { Removal }=100 \% \text { in } \\
180 \text { min }\end{array}$ & Duarte et al. (2009) \\
\hline 13. & Orange II & $\begin{array}{l}\text { a. Nanocomposites Fe sup- } \\
\text { ported on laponite clay } \\
\text { b. Nanocomposites Fe sup- } \\
\text { ported on bentonite clay }\end{array}$ & $\begin{array}{l}{[\text { Dye }]=0.2 \mathrm{mM}} \\
{[\text { Catalyst }]=1.0 \mathrm{~g} / \mathrm{L}} \\
{\left[\mathrm{H}_{2} \mathrm{O}_{2}=10 \mathrm{mM}\right.} \\
\mathrm{pH}=2.10-6.60\end{array}$ & Removal $=100 \%$ in $60 \mathrm{~min}$ & Feng et al. (2006) \\
\hline 14. & Orange II & $\begin{array}{l}\text { Plasma-synthesized hema- } \\
\text { tite and goethite }\end{array}$ & $\begin{array}{l}{[\text { Dye }]=25 \mathrm{mg} / \mathrm{L}} \\
{[\text { Catalyst }]=0.2 \mathrm{~g} / \mathrm{L}} \\
{\left[\mathrm{H}_{2} \mathrm{O}_{2}=5.0 \mathrm{mM}\right.} \\
\mathrm{pH}=3\end{array}$ & $\begin{array}{l}\text { Removal }=100 \% \text { by } \\
\text { hematite } \\
\text { Removal }=78 \% \text { by goe- } \\
\text { thite }\end{array}$ & Djowe et al. (2014) \\
\hline
\end{tabular}


Table 4 (continued)

\begin{tabular}{|c|c|c|c|c|c|}
\hline No. & Dyes & Catalyst & Reaction conditions & Results & References \\
\hline 15. & Reactive brilliant red X-3B & Fe-pillared bentonite & $\begin{array}{l}{[\text { Dye }]=10^{-4} \mathrm{~mol} / \mathrm{L}} \\
{[\text { Catalyst }]=0.5 \mathrm{~g} / \mathrm{L}} \\
{\left[\mathrm{H}_{2} \mathrm{O}_{2}\right]=10^{-2} \mathrm{~mol} / \mathrm{L}} \\
\mathrm{pH}=3.0\end{array}$ & Removal $>98 \%$ in $100 \mathrm{~min}$ & Li et al. (2006) \\
\hline 16. & Rhodamine B & $\begin{array}{l}\text { Rice hull-based silica- } \\
\text { supported iron catalyst }\end{array}$ & $\begin{array}{l}{[\text { Dye }]=5.0 \mathrm{mg} / \mathrm{L}} \\
{[\text { Catalyst }]=1.0 \mathrm{~g} / \mathrm{L}} \\
{\left[\mathrm{H}_{2} \mathrm{O}_{2}\right]=0.98 \mathrm{mmol}} \\
\mathrm{pH}=5.0\end{array}$ & Removal $=99 \%$ in $120 \mathrm{~min}$ & Gan and Li (2013) \\
\hline 17. & Rhodamine B & $\begin{array}{l}\text { Cationic cyclopentadienyl } \\
\text { iron silica }\end{array}$ & $\begin{array}{l}{[\text { Dye }]=25 \mathrm{mg} / \mathrm{L}} \\
{[\text { Catalyst }]=5.0 \mathrm{~g} / \mathrm{L}} \\
{\left[\mathrm{H}_{2} \mathrm{O}_{2}\right]=1 \mathrm{M}} \\
\mathrm{pH}=3.0\end{array}$ & Removal $=99 \%$ in $30 \mathrm{~min}$ & Chen et al. (2013) \\
\hline
\end{tabular}

and antibiotics resistance bacteria present in water have become problems of global reach (Walter and Vennes 1985).

Various oxidation methods are nowadays available to remove antibiotics from environment (Watkinson et al. 2007; Adams et al. 2002; Oturan et al. 2013; Sharma 2013; Feng et al. 2016; Anquandah et al. 2011; Gulkowska et al. 2008). Advanced oxidation process have gained much higher attention in comparison to other oxidation methods (An et al. 2010; Elmolla and Chaudhuri 2010a, b; Alaton and Dogruel 2004; Alaton et al. 2004; Kassinos et al. 2011; Zhang et al. 2006). Examples of degradation of antibiotics by different reaction processes are presented in Table 5.

Removal of amoxicillin by a mixture of $\mathrm{Fe}(\mathrm{II})$ and $\mathrm{H}_{2} \mathrm{O}_{2}$ could be observed up $100 \%$ in $\leq 90$ min when the antibiotic concentration was in the range from 10 to $500 \mathrm{mg} / \mathrm{L}$. When the level of amoxicillin was in $1 \mathrm{~g} / \mathrm{L}$, removal of the antibiotic was in hours. Degradation of amoxicillin also resulted in a decrease in TOC, generally more than $50 \%$. When photoFenton reaction using potassium ferrioxalate was applied, a decrease in toxicity of amoxicillin was seen (Table 5). Comparatively, degradation of cefradine was much less than amoxicillin under same conditions of homogeneous Fenton reaction system. Similar to amoxicillin, degradation of ampicillin and cloxacillin was up to $100 \%$, which was also in conjunction with removal of COD and TOC. When homogeneous Fenton reaction process was applied to antibiotic fermentation wastewater, significant removal of COD was observed in $60 \mathrm{~min}$ (i.e., $72.4 \%$ ).

Complete removal of ciprofloxacin by Fenton's reagent was observed in less than $2 \mathrm{~min}$ at an initial concentration of the antibiotic as $10 \mathrm{mg} / \mathrm{L}$ (Table 5). Degradation of chloramphenicol under heterogeneous Fenton system using $\mathrm{FeS}_{2}-\mathrm{H}_{2} \mathrm{O}_{2}$ at $\mathrm{pH} 8.0$ was more than $80 \%$. Chlortetracycline could be removed by homogeneous Fenton reaction processes in which a combination of Fenton with sonication gave removal of $82 \%$, a slightly higher than either Fenton reaction or sonication processes (67-76\%). Removal of fl roquinolone was low (i.e., 40\%) in use of Fenton's reagent. However, degradation of $\mathrm{fl}$ uine increased to 94\% under photo-Fenton reaction conditions. Removal of levofloxacin was sought by electro-Fenton reaction process that showed a complete elimination of the antibiotic, and a decrease in TOC was more than 50\%. Other fluoroquinolones, moxifl xacin, norfl xacin, and ofl xacin also had the complete removal by the Fenton reaction systems.

Degradation of sulfonamide antibiotics (e.g., sulfamethoxazole, sulfamethazine, sulfanilamide, and sulfasalazine, and sulfamonomethoxine) and trimethoprim has been investigated by Fenton reaction processes. Almost complete elimination of sulfonamides was observed (Table 5). Mineralizations of sulfamethoxazole and trimethoprim were also high (70-90\%). When Fenton's reagent was applied to degrade antibiotics in swine wastewater, removal of TOC was $40 \%$. Several studies have been performed on degrading tetracycline by Fenton reaction processes (Table 5). In an hour or less, removal of tetracyclines was in the range of 79-100\%, depending on the reaction conditions. In a longer time of $2 \mathrm{~h}$, mineralization up to $\sim 4 \%$ could be achieved (Table 5).

\section{Conclusion}

In the homogeneous Fenton reaction, the conditions of should be optimized in order to evaluate full potential of $\mathrm{OH}$ radicals to oxidize contaminants in water. These conditions include dosages of $\mathrm{Fe}(\mathrm{II})$ and hydrogen peroxide, $\mathrm{pH}$, and temperature. Excess concentrations of $\mathrm{Fe}(\mathrm{II})$ precipitate out as ferric hydroxide, and COD value increases by excess amount of hydrogen peroxide. Homogeneous Fenton reaction is limited to acidic $\mathrm{pH}$ that results in unwanted sludge of iron-oxy hydroxides. Heterogeneous Fenton reaction systems are being developed by applying catalysts (e.g., ferric oxides) and photolysis to enhance the eff eness to eliminate contaminants in water. However, a more research is needed on advancing application of catalysts which are 
Table 5 Treatment of antibiotics containing wastewater by different Fenton reaction processes

\begin{tabular}{|c|c|c|c|c|}
\hline S. no. & Antibiotics & Reaction conditions & Results & References \\
\hline 1. & Amoxicillin & $\begin{array}{l}\text { Box-Behnken-statistical } \\
\text { experiment design [Amoxicil- } \\
\text { lin] }=10-200 \mathrm{mg} / \mathrm{L} \\
{\left[\mathrm{H}_{2} \mathrm{O}_{2}\right]=10-500 \mathrm{mg} / \mathrm{L}} \\
{[\mathrm{Fe}(\mathrm{II})]=0-50 \mathrm{mg} / \mathrm{L} \mathrm{Fe}(\mathrm{II})}\end{array}$ & $\begin{array}{l}\text { Removal }=100 \% \text { degradation in } \\
2.5 \text { min } \\
\text { Mineralization }=37 \% \text { in } 15 \mathrm{~min}\end{array}$ & Ay and Fikret (2010) \\
\hline 2. & Amoxicillin & $\begin{array}{l}\text { Central composite factorial } \\
\text { design method [Amoxicil- } \\
\text { lin] }=450 \mu \mathrm{g} / \mathrm{L} \\
{\left[\mathrm{H}_{2} \mathrm{O}_{2}\right]=3.50-4.28 \mathrm{mg} / \mathrm{L}} \\
{[\mathrm{Fe}(\mathrm{II})]=254-350 \mu \mathrm{g} / \mathrm{L}} \\
\mathrm{pH}=3.5 \text {, tempera- } \\
\text { ture }=20-30^{\circ} \mathrm{C}\end{array}$ & Removal $=100 \%$ in $30 \mathrm{~min}$ & Homem et al. (2010) \\
\hline 3. & Amoxicillin & $\begin{array}{l}{[\text { Amoxicillin }]=10 \mathrm{mg} / \mathrm{L}} \\
{[\text { Potassium ferroxalate }]=0.1 \mathrm{~g} / \mathrm{L}} \\
\text { pH } 6.0-8.0 \\
\text { UV light irradiation }\end{array}$ & $\begin{array}{l}\text { TOC removal }=53 \% \text { in } 90 \mathrm{~min} \\
\text { Decrease in toxicity to } 65 \% \text { to } \\
5 \%\end{array}$ & Trovo et al. (2011) \\
\hline 4. & Ampicillin & $\begin{array}{l}{[\text { Ampicillin }]=20 \mathrm{mg} / \mathrm{L}} \\
{\left[\mathrm{H}_{2} \mathrm{O}_{2}\right]=400 \mu \mathrm{mol} / \mathrm{L}} \\
{[\mathrm{Fe}(\mathrm{II})]=87 \mu \mathrm{mol} / \mathrm{L} \mathrm{Fe}(\mathrm{II})} \\
\mathrm{pH}=3.5\end{array}$ & $\begin{array}{l}\text { Removal }=100 \% \text { in } 3 \mathrm{~min} \text { by } \\
\text { photo-Fenton } \\
\text { Removal }=100 \% \text { in } 10 \mathrm{~min} \text { by } \\
\text { Fenton process }\end{array}$ & Rozas et al. (2010) \\
\hline 5. & Amoxicillin, cefradine & $\begin{array}{l}{[\text { Amoxicillin }]=1 \mathrm{~g} / \mathrm{L}} \\
{\left[\mathrm{H}_{2} \mathrm{O}_{2}\right]=166.5 \mathrm{mg} / \mathrm{L}} \\
{[\mathrm{Fe}(\mathrm{II})]=166.5 \mathrm{mg} / \mathrm{L}} \\
\mathrm{pH}=3.0\end{array}$ & $\begin{array}{l}\text { Removal }(\text { amoxicillin })=97.4 \% \\
\text { in } 48 \mathrm{~h} \\
\text { Removal }(\text { cefradine })=22.5 \% \\
\text { in } 48 \mathrm{~h}\end{array}$ & Li et al. (2015) \\
\hline 6. & Amoxicillin, cloxacillin & $\begin{array}{l}{[\text { Amoxicillin] }=150 \mathrm{mg} / \mathrm{L}} \\
{[\text { Cloxacillin] }=150 \mathrm{mg} / \mathrm{L}} \\
\mathrm{H}_{2} \mathrm{O}_{2}: \mathrm{COD}=2 \\
\mathrm{Fe}(\mathrm{II}): \mathrm{H}_{2} \mathrm{O}_{2}=76 \text { at } \mathrm{pH}=3\end{array}$ & $\begin{array}{l}\text { COD removal }=79 \% \\
\text { TOC removal }=73 \% \\
\text { Ammonia-nitrogen } \\
\text { removal }=81.9 \% \text { in } 90 \mathrm{~min}\end{array}$ & Affam and Chaudhuri (2013) \\
\hline 7. & Amoxicillin, cloxacillin & $\begin{array}{l}{[\text { Amoxicillin] }=150 \mathrm{mg} / \mathrm{L}} \\
{[\text { Cloxacillin }]=150 \mathrm{mg} / \mathrm{L}} \\
{\left[\mathrm{H}_{2} \mathrm{O}_{2}\right]:[\mathrm{COD}]=2.0} \\
\text { FeGAC }=3.5 \mathrm{~g} / \mathrm{L}\end{array}$ & $\begin{array}{l}\text { COD removal }=87.5 \% \\
\text { TOC removal }=78.0 \% \\
\mathrm{NH},-\mathrm{N} \text { removal }=98.2 \% \text { in } \\
90 \text { min, } \mathrm{pH} 3.0\end{array}$ & Augstin et al. (2014) \\
\hline 8. & Amoxicillin, cloxacillin & $\begin{array}{c}{[\text { Amoxicillin }]=138 \mathrm{mg} / \mathrm{L}} \\
{[\text { Cloxacillin }]=84 \mathrm{mg} / \mathrm{L}} \\
{\left[\mathrm{H}_{2} \mathrm{O}_{2}\right] /[\mathrm{COD}]=2.5} \\
{[\mathrm{Fe}(\mathrm{II})]:\left[\mathrm{H}_{2} \mathrm{O}_{2}\right]=2.0}\end{array}$ & $\begin{array}{l}\text { COD removal }=89 \% \\
\text { Degradation }=100 \% \text { in } 1 \mathrm{~min}\end{array}$ & Elmolla and Chaudhuri (2012) \\
\hline 9. & Azithromycin, clarithromycin & $\begin{array}{l}\text { [Azithromycin]/[Clarithromy- } \\
\text { cin }]=200 \mathrm{mg} / \mathrm{L} \\
{[\mathrm{Fe}(\mathrm{II})]=0.45 \mathrm{mmol} / \mathrm{L}} \\
{\left[\mathrm{Fe}^{0}\right]=0.3 \mathrm{mmol} / \mathrm{L}} \\
{\left[\mathrm{H}_{2} \mathrm{O}_{2}\right]=0.16 / 0.3 \mathrm{mmol} / \mathrm{L}} \\
\mathrm{pH}=7.0\end{array}$ & $\begin{array}{l}\text { COD removal (azithromy- } \\
\text { cin) }=83 \% \\
\text { COD removal (clarithromy- } \\
\text { cin) }=76 \% \\
\text { Removal (azithromycin) }=90 \% \\
\text { Removal (clarithromycin) }=76 \% \\
\text { in } 1 \mathrm{~h}\end{array}$ & Mohammadi et al. (2013) \\
\hline 10. & $\begin{array}{l}\text { Amoxicillin, ampicillin, cloxacil- } \\
\text { lin }\end{array}$ & $\begin{array}{l}{[\text { Amoxicillin }]=103 \mathrm{mg} / \mathrm{L}} \\
{[\text { Ampicillin }]=104 \mathrm{mg} / \mathrm{L}} \\
{[\text { Cloxacillin }]=105 \mathrm{mg} / \mathrm{L}} \\
{[\text { Zinc oxide }]=0.5 \mathrm{~g} / \mathrm{L}} \\
\mathrm{pH}=11.0\end{array}$ & $\begin{array}{l}\text { Degradation }=100 \% \\
\text { COD removal }=23.9 \% \\
\text { TOC removal }=9.7 \% \text { in } 180 \mathrm{~min}\end{array}$ & Elmolla et al. (2010) \\
\hline 11. & $\begin{array}{l}\text { Amoxicillin, ampicillin, cloxacil- } \\
\text { lin }\end{array}$ & $\begin{array}{l}{[\text { Amoxicillin }]=104 \mathrm{mg} / \mathrm{L}} \\
{[\text { Ampicillin }]=105 \mathrm{mg} / \mathrm{L}} \\
{[\text { Cloxacillin }]=103 \mathrm{mg} / \mathrm{L}} \\
{[\mathrm{COD}] /\left[\mathrm{H}_{2} \mathrm{O}_{2}\right] /[\mathrm{Fe}(\mathrm{II})]=1: 3: 0.3} \\
\mathrm{pH}=3.0\end{array}$ & $\begin{array}{l}\text { Degradation }=100 \% \text { in } 2 \mathrm{~min} \\
\text { COD removal }=81.4 \% \\
\text { DOC removal }=54.3 \% \text { in } 60 \mathrm{~min} \\
\text { BOD } / \text { COD ratio improve- } \\
\text { ment }=10 \text { min (amoxicillin), } \\
20 \text { min (ampicillin), } 40 \mathrm{~min} \\
\text { (cloxacillin) }\end{array}$ & Elmolla and Chaudhuri (2009a) \\
\hline
\end{tabular}


Table 5 (continued)

\begin{tabular}{|c|c|c|c|c|}
\hline S. no. & Antibiotics & Reaction conditions & Results & References \\
\hline 12. & $\begin{array}{l}\text { Amoxicillin, ampicillin, cloxacil- } \\
\text { lin }\end{array}$ & $\begin{array}{l}{[\text { Amoxicillin] }=104 \mathrm{mg} / \mathrm{L}} \\
{[\text { Ampicillin] }=105 \mathrm{mg} / \mathrm{L}} \\
{[\text { Cloxacillin] }=103 \mathrm{mg} / \mathrm{L}} \\
{\left[\mathrm{H}_{2} \mathrm{O}_{2}\right]:[\mathrm{COD}]=1.5} \\
{[\mathrm{Fe}(\mathrm{II})]:\left[\mathrm{H}_{2} \mathrm{O}_{2}\right]=20} \\
\mathrm{pH}=3.0\end{array}$ & $\begin{array}{l}\text { Degradation }=100 \% \text { in } 2 \mathrm{~min} \\
\text { BOD } / \text { COD ratio improved }=0 \\
\text { to } 0.4 \\
\text { COD removal }=80.8 \% \\
\text { DOC }=58.4 \% \text { in } 50 \mathrm{~min}\end{array}$ & Elmolla and Chaudhuri (2009b) \\
\hline 14. & $\begin{array}{l}\text { Amoxicillin, ampicillin, cloxacil- } \\
\text { lin }\end{array}$ & $\begin{array}{l}{[\text { Amoxicillin] }=100 \mathrm{mg} / \mathrm{L}} \\
{[\text { Ampicillin }]=220 \mathrm{mg} / \mathrm{L}} \\
{[\text { Cloxacillin }]=500 \mathrm{mg} / \mathrm{L}} \\
{\left[\mathrm{H}_{2} \mathrm{O}_{2}\right]:[\mathrm{COD}]=3.0} \\
\mathrm{pH}=3.0\end{array}$ & $\begin{array}{l}\text { COD removal (all three antibiot- } \\
\text { ics) }=\sim 80 \%\end{array}$ & Elmolla et al. (2010) \\
\hline 15. & $\begin{array}{l}\text { Antibiotic fermentation waste- } \\
\text { water }\end{array}$ & $\begin{array}{l}\mathrm{COD}=3279 \mathrm{mg} / \mathrm{L} \\
\text { TOC }=1296.3 \mathrm{mg} / \mathrm{L} \\
\text { Color }=3000 \\
{\left[\mathrm{H}_{2} \mathrm{O}_{2}\right]=150 \mathrm{mg} / \mathrm{L}} \\
{[\mathrm{Fe}(\mathrm{II})]=120 \mathrm{mg} / \mathrm{L}} \\
\mathrm{pH}=4.0\end{array}$ & $\begin{array}{l}\text { Color removal }=66.6 \% \\
\text { COD removal }=72.4 \% \text { after } \\
60 \text { min }\end{array}$ & Xing and Sun (2009) \\
\hline 17. & Ciprofloxacin & $\begin{array}{l}{[\text { Ciprofloxacin }]=10 \mathrm{mg} / \mathrm{L}} \\
{\left[\mathrm{H}_{2} \mathrm{O}_{2}\right]=2.5 \mathrm{mM}} \\
{[\mathrm{Fe}(\mathrm{II})]=2.0 \mathrm{mg} / \mathrm{L}} \\
\mathrm{pH}=2.8\end{array}$ & Degradation $=80 \%$ in $1.8 \mathrm{~min}$ & Lima et al. (2014) \\
\hline 18. & Cefalexin & $\begin{array}{l}{[\text { Cefalexin }]=200 \mathrm{mg} / \mathrm{L}} \\
{[\mathrm{Fe}(\mathrm{II})]=1 \mathrm{mM}} \\
\mathrm{pH}=3.0 \\
\text { Current density }=6.66 \mathrm{~mA} / \mathrm{cm}^{2}\end{array}$ & $\begin{array}{l}\text { TOC removal }=49 \% \\
\text { Mineralization }=100 \%\end{array}$ & Estrada et al. (2012) \\
\hline 19. & Chloramphenicol & $\begin{array}{l}{[\text { Chloramphenicol] }=50 \mathrm{mg} / \mathrm{L}} \\
{[\mathrm{GLDA}]=100 \mu \mathrm{mol} / \mathrm{L}} \\
{\left[\mathrm{FeS}_{2}\right]=100 \mathrm{mg} / \mathrm{L}} \\
{\left[\mathrm{H}_{2} \mathrm{O}_{2}\right]=1 \mathrm{mmol} / \mathrm{L}} \\
\mathrm{pH}=8.0\end{array}$ & Degradation $=83.3 \%$ & Wu et al. (2016) \\
\hline 20. & Chlortetracycline & $\begin{array}{l}{[\text { Sludge }]=34 \mathrm{~g} / \mathrm{L}} \\
{[\mathrm{Fe}(\mathrm{II})]=30 \mathrm{mg} / \mathrm{L}} \\
{\left[\mathrm{H}_{2} \mathrm{O}_{2}\right]=30 \mathrm{mg} / \mathrm{L}} \\
\mathrm{pH}=3.0\end{array}$ & $\begin{array}{l}\text { Removal (ultrasonication pro- } \\
\text { cess) }=67 \% \\
\text { Removal (fenton oxidation pro- } \\
\text { cess) }=76 \% \\
\text { Removal (ferro-sonica- } \\
\text { tion) }=82 \%\end{array}$ & Pulicharla et al. (2017) \\
\hline 21. & $\begin{array}{l}\text { Ciprofloxacin, sulphamethoxa- } \\
\text { zole, trimethoprim }\end{array}$ & $\begin{array}{l}{[\text { Ciprofloxacin] }] /[\mathrm{sul}-} \\
\text { phamethoxazole]/[Tri- } \\
\text { methoprim] }=4.24 \times 10^{-2} \\
\mathrm{mg}_{\mathrm{c}} \mathrm{m}^{2} \mathrm{~W}^{-2} \mathrm{~L}^{-1} \\
{\left[\mathrm{H}_{2} \mathrm{O}_{2}\right]=2.5 \mathrm{mM}} \\
\mathrm{pH}=2.8\end{array}$ & $\begin{array}{l}\text { Removal }=\sim 60 \% \text { removal after } \\
240 \mathrm{~min}\end{array}$ & Lima et al. (2017) \\
\hline 22. & $\begin{array}{l}\text { Clarithromycin, sulfamethoxa- } \\
\text { zole }\end{array}$ & $\begin{array}{l}{[\text { Clarithromycin }] /[\text { sulfamethoxa- }} \\
\text { zole }]=100 \mathrm{ppb} \\
{\left[\mathrm{H}_{2} \mathrm{O}_{2}\right]=50 \mathrm{mg} / \mathrm{L}} \\
{[\mathrm{Fe}(\mathrm{III})]=5 \mathrm{mg} / \mathrm{L}} \\
\mathrm{pH}=4.0\end{array}$ & $\begin{array}{l}\text { Removal (clarithromycin) }=70 \% \\
\text { Removal (sulfamethoxa- } \\
\text { zole })=95 \%\end{array}$ & Karaolia et al. (2014) \\
\hline 23. & $\begin{array}{l}\text { Chloramphenicol, ciprofloxacin, } \\
\text { dipyrone }\end{array}$ & $\begin{array}{l}{[\text { Chloramphenicol] } /[\text { ciprofloxa- }} \\
\text { cin }] /[\text { dipyrone }]=0.15 \mathrm{mM} \\
{\left[\mathrm{H}_{2} \mathrm{O}_{2}\right]=22.5 \mathrm{mM}} \\
{[\mathrm{Fe}(\mathrm{II})]=2.25 \mathrm{mM}} \\
\mathrm{pH}=3.5\end{array}$ & $\begin{array}{l}\text { Removal (chlorampheni- } \\
\quad \text { col) }=52 \% \\
\text { Removal (ciprofloxacin) }=42 \% \\
\text { Removal (dipyrone) }=47 \% \text { in } \\
45 \text { min }\end{array}$ & Giri and Golder (2015) \\
\hline 24. & Enoxacin & $\begin{array}{l}{[\text { Enoxacin }]=50 \mathrm{mg} / \mathrm{L}} \\
{[\mathrm{Fe}(\mathrm{II})]=0.2 \mathrm{mmol} / \mathrm{L}} \\
\text { Current intensity }=300 \mathrm{~mA}\end{array}$ & $\begin{array}{l}\text { Residual yields }=54 \% \text { and } 43 \% \\
\text { after } 120 \mathrm{~min} \\
\text { Fluorine }=77 \%, \text { nitrate }=5 \%, \\
\text { ammonia }=24 \% \text { after } 180 \mathrm{~min}\end{array}$ & Annabi et al. (2016) \\
\hline 25. & Flumequine & $\begin{array}{l}\text { [Flumequine }]=500 \mu \mathrm{g} / \mathrm{L} \\
{[\mathrm{Fe}(\mathrm{II})]=0.5 \mathrm{mmol} / \mathrm{L}} \\
{\left[\mathrm{H}_{2} \mathrm{O}_{2}\right]=2.0 \mathrm{mmol} / \mathrm{L}}\end{array}$ & $\begin{array}{l}\text { Degradation }(\text { Fenton })=40 \% \\
\text { Degradation (photo-Fen- } \\
\text { ton) }=94 \% \text { after } 60 \mathrm{~min}\end{array}$ & Silva et al. (2013) \\
\hline
\end{tabular}


Table 5 (continued)

\begin{tabular}{|c|c|c|c|c|}
\hline S. no. & Antibiotics & Reaction conditions & Results & References \\
\hline 26. & Levofloxacin & $\begin{array}{l}{[\text { Levofloxacin }]=200 \mathrm{mg} / \mathrm{L}} \\
{\left[\mathrm{Na}_{2} \mathrm{SO}_{4}\right]=0.05 \mathrm{M}} \\
{[\mathrm{Fe}(\mathrm{II})]=1 \mathrm{mM}} \\
\mathrm{pH}=3.0 \\
\text { Current inten- } \\
\quad \text { sity }=6.67 \mathrm{~mA} \mathrm{~cm}^{-2}\end{array}$ & $\begin{array}{l}\text { Removal }=100 \% \text { in } 120 \mathrm{~min} \\
\text { TOC removal }=60 \% \text { after } \\
360 \text { min }\end{array}$ & Gong et al. (2016) \\
\hline 27. & Levofloxacin & $\begin{array}{l}{[\text { Levofloxacin }]=0.23 \mathrm{mM}} \\
{\left[\mathrm{Na}_{2} \mathrm{SO}_{4}\right]=0.05 \mathrm{M}} \\
{[\mathrm{Fe}(\mathrm{II})]=0.2 \mathrm{mM}} \\
\mathrm{pH}=3.0 \\
\text { Current intensity }=300 \mathrm{~mA}\end{array}$ & $\begin{array}{l}\text { Mineralization }=100 \% \\
\mathrm{TOC}=95 \% \text { for } 8 \mathrm{~h}\end{array}$ & Barhoumi et al. (2015) \\
\hline 28. & Moxifloxacin & $\begin{array}{l}{[\text { Moxifloxacin }]=0.15 \mathrm{mM}} \\
{[\mathrm{Fe}(\mathrm{II})]=0.5 \mathrm{mM}} \\
\mathrm{pH}=3.0\end{array}$ & $\begin{array}{l}\text { Several intermediates were } \\
\text { formed for the degradation of } \\
\text { moxifloxacin, which was con- } \\
\text { firmed by LC-MS analysis }\end{array}$ & Yahya et al. (2017) \\
\hline 29. & Norfloxacin & $\begin{array}{l}{[\text { Norfloxacin }]=100 \mathrm{mg} / \mathrm{L}} \\
{\left[\mathrm{Fe}_{0}\right]=100 \mathrm{mg} / \mathrm{L}} \\
{\left[\mathrm{H}_{2} \mathrm{O}_{2}\right]=10 \mathrm{mmol} / \mathrm{L}} \\
\mathrm{pH}=4.0\end{array}$ & $\begin{array}{l}\text { Degradation }=95 \% \text { within } \\
40 \text { min } \\
\text { Mineralization }=50 \%\end{array}$ & Zhang et al. (2017) \\
\hline 30. & Norfloxacin & $\begin{array}{l}{[\text { Norfloxacin }]=0.25 \mathrm{mM}} \\
{\left[\mathrm{Na}_{2} \mathrm{SO}_{4}\right]=0.05 \mathrm{M}} \\
{[\mathrm{Fe}(\mathrm{III})]=0.1 \mathrm{mM}} \\
\mathrm{pH}=3.0 \\
\text { Current intensity }=60 \mathrm{~mA}\end{array}$ & Mineralization $=97.7 \%$ after $5 \mathrm{~h}$ & Özcan et al. (2016) \\
\hline 31. & Norfloxacin & $\begin{array}{l}{[\text { Norfloxacin }]=15 \mathrm{mg} / \mathrm{L}} \\
{\left[\mathrm{H}_{2} \mathrm{O}_{2}\right]=2.1 \mathrm{mmol} / \mathrm{L}} \\
\mathrm{pH}=7.0\end{array}$ & $\begin{array}{l}\text { Degradation }=100 \% \text { degradation } \\
\text { Mineralization }=55 \% \text { minerali- } \\
\text { zation }\end{array}$ & Santos et al. (2015) \\
\hline 32. & Ofloxacin & $\begin{array}{l}\left(\mathrm{UV} / \mathrm{H}_{2} \mathrm{O}_{2}\right) \\
{[\mathrm{Ofloxacin}]=10 \mathrm{mg} / \mathrm{L}} \\
{[\mathrm{Fe}(\mathrm{II})]=2 \mathrm{mg} / \mathrm{L}} \\
{\left[\mathrm{H}_{2} \mathrm{O}_{2}\right]=2.5 \mathrm{mg} / \mathrm{L}}\end{array}$ & Degradation $=100 \%$ & Michael et al. (2013) \\
\hline 33. & Oxacillin & $\begin{array}{l}{[\text { Oxacillin }]=203 \mu \mathrm{mol} / \mathrm{L}} \\
{[\mathrm{Fe}(\mathrm{II})]=90 \mu \mathrm{mol} / \mathrm{L}} \\
{\left[\mathrm{H}_{2} \mathrm{O}_{2}\right]=10 \mu \mathrm{mol} / \mathrm{L}}\end{array}$ & $\begin{array}{l}\text { Mineralization }=100 \% \text { after } \\
5 \text { min }\end{array}$ & Giraldo-Aguirre et al. (2017) \\
\hline 34. & Ofloxacin, trimethoprim & $\begin{array}{l}{[\text { Ofloxacin }] /[\text { trimetho- }} \\
\text { prim }]=100 \mu \mathrm{g} / \mathrm{L} \\
{[\mathrm{Fe}(\mathrm{II})]=5 \mathrm{mg} / \mathrm{L}}\end{array}$ & Removal $=100 \%$ & Michael et al. (2012) \\
\hline 35. & Sulfasalazine & $\begin{array}{l}{\left[\mathrm{H}_{2} \mathrm{O}_{2}\right]=75 \mathrm{mg} / \mathrm{L} \text { in solar light }} \\
{[\text { Sulfasalazine }]=100 \mathrm{mg} / \mathrm{L}} \\
{[\mathrm{Fe}(\mathrm{II})]=0.20 \mathrm{mM}}\end{array}$ & $\begin{array}{l}\text { Removal }=\sim 99.5 \% \\
\text { TOC removal }=82.4 \%\end{array}$ & Fan et al. (2011) \\
\hline 36. & Sulfamethoxazole & $\begin{array}{l}{\left[\mathrm{H}_{2} \mathrm{O}_{2}\right]=16 \mathrm{mM}} \\
{[\mathrm{Sulfamethoxazole}]=200 \mathrm{mg} / \mathrm{L}} \\
{[\mathrm{Fe}(\mathrm{II})]=1 \mathrm{mM}} \\
\mathrm{pH}=3.0\end{array}$ & $\begin{array}{l}\text { COD removal }=41 \% \text { in } 60 \text { min } \\
\text { TOC removal }=80 \% \text { (photo-elec- } \\
\text { tro-Fenton process) in } 6 \mathrm{~h} \\
\text { Mineralization }=63 \% \text { (electro- } \\
\text { Fenton process) } \\
\text { TOC removal }=25 \% \text { (electro- } \\
\text { Fenton process) }\end{array}$ & Wang et al. (2011) \\
\hline 37. & Sulfamethoxazole & $\begin{array}{l}{[\text { Sulfamethoxazole }]=50 \mathrm{mg} / \mathrm{L}} \\
{[\mathrm{Fe}(\mathrm{II})=2.6 \mathrm{mg} / \mathrm{L}}\end{array}$ & Toxicity reduction $=80 \%$ to $20 \%$ & Trovo et al. (2009) \\
\hline 38. & Sulfamethoxazole & $\begin{array}{l}{\left[\mathrm{H}_{2} \mathrm{O}_{2}\right]=120 \mathrm{mg} / \mathrm{L}} \\
{[\text { Sulfamethoxazole }]=200 \mathrm{mg} / \mathrm{L}} \\
{[\mathrm{Fe}(\mathrm{II})]=10 \mathrm{mg} / \mathrm{L}} \\
{\left[\mathrm{H}_{2} \mathrm{O}_{2}\right]=300 \mathrm{mg} / \mathrm{L}} \\
\mathrm{pH}=2.8\end{array}$ & Removal $=100 \%$ & Gonzalez et al. (2007) \\
\hline 39. & Sulfamethazine & $\begin{array}{l}{[\text { Sulfamethazine }]=50 \mathrm{mg} / \mathrm{L}} \\
{[\mathrm{Fe}(\mathrm{II})]=40 \mathrm{mg} / \mathrm{L}} \\
{\left[\mathrm{H}_{2} \mathrm{O}_{2}\right]=600 \mathrm{mg} / \mathrm{L}}\end{array}$ & Degradation $=100 \%$ in $2 \mathrm{~min}$ & Moya et al. (2010) \\
\hline
\end{tabular}


Table 5 (continued)

\begin{tabular}{|c|c|c|c|c|}
\hline S. no. & Antibiotics & Reaction conditions & Results & References \\
\hline 40. & Sulfamethazine & $\begin{array}{l}{[\text { Sulfamethazine }]=20 \mathrm{mg} / \mathrm{L}} \\
{[\mathrm{Fe}(\mathrm{II})]=3.5-28 \mathrm{mg} / \mathrm{L}} \\
{\left[\mathrm{H}_{2} \mathrm{O}_{2}\right]=10-400 \mathrm{mg} / \mathrm{L}} \\
\text { Current intensity }=2 \mathrm{mWCm}^{-2}\end{array}$ & $\begin{array}{l}\text { Mineralization }=70 \% \text { after } \\
120 \text { min }\end{array}$ & Kitsiou et al. (2014) \\
\hline 41. & Sulfamethazine & $\begin{array}{l}{[\text { Sulfamethazine }]=20 \mathrm{mg} / \mathrm{L}} \\
{[\mathrm{Ce}-\mathrm{Fe} \text {-graphene }]=0.5 \mathrm{~g} / \mathrm{L}} \\
{\left[\mathrm{H}_{2} \mathrm{O}_{2}\right]=8 \mathrm{mM}} \\
\mathrm{pH}=7.0\end{array}$ & $\begin{array}{l}\text { Degradation }=99 \% \\
\text { TOC removal }=73 \%\end{array}$ & Wan et al. (2016) \\
\hline 42. & Sulfanilamide & $\begin{array}{l}{[\text { Sulfanilamide }]=0.6 \mathrm{mM}} \\
{\left[\mathrm{Na}_{2} \mathrm{SO}_{4}\right]=0.05 \mathrm{M}} \\
{[\mathrm{Fe}(\mathrm{II})]=0.20 \mathrm{mM}} \\
\mathrm{pH}=3.0 \\
\text { Current intensity }=300 \mathrm{~mA}\end{array}$ & Mineralization $=100 \%$ & Ghenymy et al. (2014) \\
\hline 43. & Sulfamonomethoxine & $\begin{array}{l}{[\text { Sulfamonomethox- }} \\
\text { ine }]=4.53 \mathrm{mg} / \mathrm{L} \\
{\left[\mathrm{H}_{2} \mathrm{O}_{2}\right]=0.49 \mathrm{mmol} / \mathrm{L}} \\
{[\mathrm{Fe}(\mathrm{II})]=19.51 \mu \mathrm{mol} / \mathrm{L}} \\
\mathrm{pH}=4.0\end{array}$ & $\begin{array}{l}\text { Degradation }=98.5 \% \\
\text { COD removal }=99 \% \text { after } \\
120 \text { min }\end{array}$ & Hui et al. (2012) \\
\hline 44. & Sulfonamide & $\begin{array}{l}{[\text { Sulfonamide }]=0.19 \mathrm{mM}} \\
\mathrm{Fe}(\mathrm{II}): \mathrm{H}_{2} \mathrm{O}_{2}=1.5 \\
\mathrm{pH}=2.5\end{array}$ & $\begin{array}{l}\text { Degradation }=99.9 \% \\
\text { COD removal } 64.7-70.7 \% \text { in } \\
15 \text { min }\end{array}$ & Dehghani et al. (2013) \\
\hline 45. & Sulfamethoxazole, trimethoprim & $\begin{array}{l}\text { [Sulfamethoxazole }] /[\text { trimetho- } \\
\text { prim }]=20 \mathrm{mg} / \mathrm{L} \\
{\left[\mathrm{H}_{2} \mathrm{O}_{2}\right] \text { (sulfamethoxa- }} \\
\text { zole })=2.6 \mathrm{mM} \\
{\left[\mathrm{H}_{2} \mathrm{O}_{2}\right] \text { (trimethoprim) }=3.0 \mathrm{mM}} \\
{[\mathrm{Fe}(\mathrm{III})]=0.5 \mathrm{mg} / \mathrm{L}} \\
\mathrm{pH}=5.0\end{array}$ & $\begin{array}{l}\text { Mineralization (sulfamethoxa- } \\
\text { zole) }=70 \% \\
\text { Mineralization (trimetho- } \\
\text { prim) }=90 \%\end{array}$ & Dias et al. (2014) \\
\hline 46. & $\begin{array}{l}\text { Sulfamethoxazole, erythromycin, } \\
\text { clarithromycin }\end{array}$ & $\begin{array}{l}{[\text { Substrate }]=100 \mu \mathrm{g} / \mathrm{L}} \\
{[\mathrm{Fe}(\mathrm{III})]=5 \mathrm{mg} / \mathrm{L}} \\
{\left[\mathrm{H}_{2} \mathrm{O}_{2}\right]=50 \mathrm{mg} / \mathrm{L}} \\
\mathrm{pH}=4.0\end{array}$ & Removal $=100 \%$ & Karaolia et al. (2017) \\
\hline 47. & $\begin{array}{l}\text { Swine wastewater }(5 \text { sulfona- } \\
\text { mide }+1 \text { macrolide }\end{array}$ & $\begin{array}{l}{[\text { Antibiotics }]=1 \mathrm{mg} / \mathrm{L}} \\
{[\mathrm{Fe}(\mathrm{II})]=0.91 \mathrm{mmol} / \mathrm{L},} \\
{\left[\mathrm{H}_{2} \mathrm{O}_{2}\right]=1.37 \mathrm{mmol} / \mathrm{L}} \\
{[\mathrm{Fe}(\mathrm{II})]:\left[\mathrm{H}_{2} \mathrm{O}_{2}\right]=0.66, \mathrm{pH}=5.0}\end{array}$ & $\begin{array}{l}\text { TOC removal }=40 \% \\
\text { As removal }=78 \%, \mathrm{Cu} \\
\text { removal }=36 \% \\
\mathrm{~Pb} \text { removal }=18 \% \text {, Toxicity } \\
\text { removal }=25 \%\end{array}$ & Ben et al. (2009) \\
\hline 48. & Tetracycline & $\begin{array}{l}{[\text { Tetracycline }]=20 \mathrm{mg} / \mathrm{L}} \\
{\left[\mathrm{Fe}_{3} \mathrm{O}_{4}\right.} \\
\mathrm{C}]=0.15 \mathrm{~g} / \mathrm{L},\left[\mathrm{H}_{2} \mathrm{O}_{2}\right]=3 \mathrm{mM} \\
\mathrm{pH}=3.0\end{array}$ & $\begin{array}{l}\text { Removal }=79 \% \text { in } 44 \mathrm{~min} \\
\text { Mineralization }=43.7 \% \text { in } \\
120 \mathrm{~min}\end{array}$ & Kakavandi et al. (2016) \\
\hline 49. & Tetracycline & $\begin{array}{l}{[\text { Tetracycline }]=100 \mathrm{mg} / \mathrm{L}} \\
{[\mathrm{Fe}(\mathrm{II})]=1.0 \mathrm{~g} / \mathrm{L}} \\
{\left[\mathrm{H}_{2} \mathrm{O}_{2}\right]=150 \mathrm{mmol} / \mathrm{L}} \\
\mathrm{pH}=3.7\end{array}$ & $\begin{array}{l}\text { Removal }=93.6 \% \text { in } 60 \text { min } \\
\text { Mineralization }=31.8 \% \text { after } \\
60 \text { min }\end{array}$ & Hou et al. (2016) \\
\hline 50. & Tetracycline & $\begin{array}{l}{[\text { Tetracycline }]=40 \mathrm{mg} / \mathrm{L}} \\
{\left[\mathrm{Fe}_{3} \mathrm{O}_{4}\right.} \\
\text { void } \\
\left.\mathrm{TiO}_{2}\right]=0.25 \mathrm{~g} / \mathrm{L} \\
{\left[\mathrm{H}_{2} \mathrm{O}_{2}\right]=0.377 \mathrm{M}, \mathrm{pH}=3.0,9.0}\end{array}$ & $\begin{array}{l}\text { Degradation }=100 \% \text { at } \mathrm{pH} 3.0 \\
\text { Degradation }=75 \% \text { at } \mathrm{pH} 9 \\
\text { within } 6 \mathrm{~min}\end{array}$ & Du et al. (2017) \\
\hline 51 & Tetracycline & $\begin{array}{l}{[\text { Tetracycline }]=40 \mathrm{mg} / \mathrm{L}} \\
{[\mathrm{Fe}(\mathrm{II})]=5 \mathrm{mg} / \mathrm{L}} \\
{\left[\mathrm{H}_{2} \mathrm{O}_{2}\right]=71.5 \mathrm{mg} / \mathrm{L}}\end{array}$ & Mineralization $=100 \%$ & Turbay et al. (2013) \\
\hline
\end{tabular}


applicable in a wide range of $\mathrm{pH}$ and can recycle iron in the Fenton reaction system.

Fenton oxidation process can efficiently remove a range of contaminants in water. For example, antibiotics such as ofl xacin, trimethoprim, sulfonamide and sulfamethoxazole could be completely using the Fenton reaction system. However, Fenton reactions process needs to be combined with other methods to achieve thorough mineralization. Significantly, most of the studies in the literature on removing contaminants by Fenton reaction processes are on the bench scale, and a very little is known on performing at a pilot scale with polluted water. It is important Fenton reaction system should be demonstrated by using contaminated water containing nitrate, bromide, and natural organic matter. These constituents of water scavenge $\mathrm{OH}$ radicals and thus decrease the eff eness of the advanced oxidation processes. Furthermore, pilot and field scale testing of the selected Fenton reaction is required to demonstrate the capabilities, possible limitation, and reaction conditions of Fenton reaction processes to produce drinking water from contaminated source water.

Acknowledgements Dr. Bhawana Jain (postdoctoral fellow, No. F.151/2013-14/PDFWM-2013-14-GE-CHH-18784 (SA-II)) is thankful to UGC, Delhi, India, for Research Project grants. Professor Hyunook Kim appreciates the financial support by Korea Ministry of Environment (MOE) (Project ID: 2015001790002). We thank reviewers for their comments, which improved this paper greatly.

\section{References}

Acisli O, Khataee A, Karaca S, Karimi A, Dogan E (2017) Combination of ultrasonic and Fenton processes in the presence of magnetite nanostructures prepared by high energy planetary ball mill. Ultrason Sonochem 34:754-762. https://doi.org/10.1016/j.ultso nch.2016.07.011

Adams C, Wang Y, Loftin K, Meyer M (2002) Removal of antibiotics from surface and distilled water in conventional water treatment processes. J Environ Eng 128:253-260. https://doi.org/10.1061/ (ASCE)0733-9372(2002)128:3(253)

Affam AC, Chaudhuri M (2013) Optimization of Fenton treatment of amoxicillin and cloxacillin antibiotics in aqueous solution. Desalin Water Treat 52:1878-1884. https://doi.org/10.1080/19443 994.2013.794015

Alalm MG, Tawfik A, Ookawara S (2015) Comparison of solar $\mathrm{TiO}_{2}$ photocatalysis and solar photo-Fenton for treatment of pesticides industry wastewater: operational conditions, kinetics and costs. J Water Process Eng 8:55-63. https://doi.org/10.1016/j. jwpe.2015.09.007

Alaton IA, Dogruel S (2004) Pretreatment of penicillin formulation effl by advanced oxidation processes. J Hazard Mater 112:105-113. https://doi.org/10.1016/j.jhazmat.2004.04.009

Alaton IA, Dogruel S, Baykal E, Gerone G (2004) Combined chemical and biological oxidation of penicillin formulation effl J Environ Manag 73:155-163. https://doi.org/10.1016/j.jenvm an.2004.06.007

Aljuboury DDA, Palaniandy P, Abdul Aziz HB, Feroz S, Abu Amr SA (2017) Performance of the photocatalyst and Fenton processes to treat the petroleum wastewater-a review. Glob Nest J 19(3):396-411

An T, Yang H, Li G, Song W, Cooper WJ, Nie X (2010) Kinetics and mechanism of advanced oxidation processes (AOPs) in degradation of ciprofl xacin. Appl Catal B Environ 94:288-294. https://doi.org/10.1016/j.apcatb.2009.12.002

Annabi C, Fourcade F, Soutrel I, Geneste F, Floner D, Bellakhal N, Amrane A (2016) Degradation of enoxacin antibiotic by the electro-Fenton process: optimization, biodegradability improvement and degradation mechanism. J Environ Manag 165(1):96-105. https://doi.org/10.1016/j.jenvman.2015.09.018

Anquandah GAK, Sharma VK, Knight DA, Batchu SR, Gardinali PR (2011) Oxidation of trimethoprim by ferrate(VI): kinetics, products and antibacterial activity. Environ Sci Technol 45(24):10575-10581. https://doi.org/10.1021/es202237g

Anumol T, Dagnino S, Vandervort DR, Snyder SA (2016) Transformation of polyfl inated compounds in natural waters by advanced oxidation processes. Chemosphere 144:1780-1787. https://doi.org/10.1016/j.chemosphere.2015.10.070

Augstin CA, Chaudhuri M, Mohammed K, Shamsul R (2014) Optimization of modifi d Fenton $\left(\mathrm{FeGAC} / \mathrm{H}_{2} \mathrm{O}_{2}\right)$ pretreatment of antibiotics. Pert J Sci Technol 22(1):239-254. https://doi. org/10.1080/19443994.2013.794015

Ay F, Fikret K (2010) Advanced oxidation of amoxicillin by Fenton's reagent treatment. J Hazard Mater 179:622-627. https://doi. org/10.1016/j.jhazmat.2010.03.048

Badawy MI, Wahaab RA, EI-Kalliny AS (2009) Fenton biological treatment for the removal of some pharmaceuticals from industrial wastewater. J Hazard Mater 167:567-574. https:// doi.org/10.1016/j.jhazmat.2009.01.023

Bakker K (2012) Water security: research challenges and opportunities. Science 337:914-915. https://doi.org/10.1126/scien ce. 1226337

Barbosa MO, Moreira NFF, Ribeiro AR, Pereira MFR, Silva AMT (2016) Occurrence and removal of organic micropollutants: an overview of the watch list of EU Decision 2015/495. Water Res 94:257-279. https://doi.org/10.1016/j.watres.2016.02.047

Barbusinski K (2005) Toxicity of industrial waste water treated by Fenton's reagent. Pol J Environ Stud 14:11-16

Barbusinski K, Filipek K (2001) Toxicity of industrial waste water treated by Fenton's reagent. Pol J Environ Stud 10:207-212

Barhoumi N, Labiadh L, Oturan MA, Oturan N, Gadri A, Ammar S, Brillas E (2015) Electrochemical mineralization of the antibiotic levofl xacin by electro-Fenton-pyrite process. Chemosphere 141:250-257. https://doi.org/10.1016/j.chemospher e.2015.08.003

Barhoumi N, Oturan N, Olvera-Vargas H, Brillas E, Gadri A, Ammar S, Oturan MA (2016) Pyrite as a sustainable catalyst in electroFenton process for improving oxidation of sulfamethazine. Kinetics, mechanism and toxicity assessment. Water Res 94:52-61. https://doi.org/10.1016/j.watres.2016.02.042

Bataineh H, Pestovsky O, Bakac A (2012) pH-induced mechanistic changeover from hydroxyl radicals to iron(IV) in the Fenton reaction. Chem Sci 3:1594-1599. https://doi.org/10.1039/c2sc20099f

Bautista P, Mohedano AF, Casas JA, Zazo JA, Rodriguez JJ (2008) An overview of the application of Fenton oxidation to industrial wastewaters treatment. J Chem Technol Biotechnol 83:13231338. https://doi.org/10.1002/jctb.1988

Ben W, Quiang Z, Pan X, Chen M (2009) Removal of veterinary antibiotics from sequencing batch reactor (SBR) pretreated swine wastewater by Fenton's reagent. Water Res 43:4392-4402. https ://doi.org/10.1016/j.watres.2009.06.057

Benatti CT, Tavares CRG (2012) Fenton's process for the treatment of mixed waste chemicals. In: Organic pollutants ten years after the Stockholm convention-Environmental and analytical update, $\mathrm{pp}$ 247-270. https://doi.org/10.5772/31225 
Bielski BHJ, Richter HW (1977) A study of the superoxide radical chemistry by stopped-flow radiolysis and radiation induced oxygen consumption. J Am Chem Soc 99(9):3019-3023. https ://doi.org/10.1021/ja00451a028

Blum KM, Andersson PL, Ahrens L, Wiberg K, Haglund P (2018) Persistence, mobility and bioavailability of emerging organic contaminants discharged from sewage treatment plants. Sci Total Environ 612:1532-1542. https://doi.org/10.1016/j.scito tenv.2017.09.006

Bocos E, Oturan N, Pazos M, Sanromán MÁ, Oturan MA (2016) Elimination of radio contrast agent diatrizoic acid by photoFenton process and enhanced treatment by coupling with electro-Fenton process. Environ Sci Pollut Res 23(19):1913419144. https://doi.org/10.1007/s11356-016-7054-x

Boczkaj G, Fernandes A (2017) Wastewater treatment by means of advanced oxidation processes at basic $\mathrm{pH}$ conditions: a review. Chem Eng J 320:608-633. https://doi.org/10.1016/j. cej.2017.03.084

Bouafia-Chergui S, Oturan N, Khalaf H, Oturan MA (2010) Parametric study on the eff of the ratios $\left[\mathrm{H}_{2} \mathrm{O}_{2}\right] /\left[\mathrm{Fe}^{3+}\right]$ and $\left[\mathrm{H}_{2} \mathrm{O}_{2}\right] /$ [substrate] on the photo-Fenton degradation of cationic azo dye basic blue 41. J Environ Sci Health A Tox Hazard Subst Environ Eng 45(5):622-629. https://doi.org/10.1080/10934 521003595746

Brillas E, Martínez-Huitle CA (2015) Decontamination of wastewaters containing synthetic organic dyes by electrochemical methods: an updated review. Appl Catal B 166-167:603-643. https://doi. org/10.1016/j.apcatb.2014.11.016

Brillas E, Sires I, Oturan MA (2009) Electro-Fenton process and related electrochemical technologies based on Fenton's reaction chemistry. Chem Rev 109:6570-6631. https://doi.org/10.1021/ cr900136g

Brown KD, Kulis J, Thomson B, Chapman TH, Mawhinney DB (2006) Occurrence of antibiotics in hospital, residential, and dairy effluent, municipal wastewater and Rio Grande in Mexico. Sci Total Environ 366:772-783. https://doi.org/10.1016/j.scito tenv.2005.10.007

Cai C, Zhang Z, Liu J, Shan N, Zhang H, Dionysiou DD (2016) Visible light-assisted heterogeneous Fenton with $\mathrm{ZnFe}_{2} \mathrm{O}_{4}$ for the degradation of orange II in water. Appl Catal B Environ. https:// doi.org/10.1016/j.apcatb.2015.09.056

Cao M, Wang L, Wang L, Chen J, Lu X (2013) Remediation of DDTs contaminated soil in a novel Fenton-like system with zero-valent iron. Chemosphere 90(8):2303-2308. https://doi.org/10.1016/j. chemosphere.2012.09.098

Chakma S, Moholkar VS (2014) Investigations in synergism of hybrid advanced oxidation processes with combinations of sonolysis + fenton process + UV for degradation of bisphenol A. Ind Eng Chem Res 53(16):6855-6865. https://doi.org/10.1021/ie500 474f

Chakma S, Moholkar VS (2015) Intensifi of wastewater treatment using sono-hybrid processes: an overview of mechanistic synergism. Indian Chem Eng 57:359-381. https://doi. org/10.1080/00194506.2015.1026948

Chen Y, Jin S, Liu J, Zhao B, Wang T (2013) Photo-Fenton reaction of supported cationic cyclopentadienyl iron complexes of aren and application as heterogeneous catalysts in photodegradation of dyes under visible light. Inorganic Chimie Acta 406:37-43. https://doi.org/10.1016/j.ica.2013.07.005

Chen Q, Wu P, Li Y, Zhu N, Dang Z (2009) Heterogeneous photo-Fenton degradation of reactive brilliant orange X-GN over iron pillared montmorillonite under visible irradiation. J Hazard Mater 168:901-908. https://doi.org/10.1016/j.jhazmat.2009.02.107

Cheng M, Zeng G, Huang D, Lai C, Xu P, Zhang C, Liu Y (2016) Hydroxyl radicals based advanced oxidation processes (AOPs) for remediation of soils contaminated with organic compounds: a review. Chem Eng J 284:582-598. https://doi.org/10.1016/j. cej.2015.09.001

Cizmas L, Sharma VK, Gray CM, Mcdonald TJ (2015) Pharmaceuticals and personal care products in water: occurrence, toxicity and risk. Environ Chem Lett 13(4):381-394. https://doi.org/10.1007/ s10311-015-0524

Clarizia L, Russo D, Di Somma I, Marotta R, Andreozzi R (2017) Homogeneous photo-Fenton processes at near neutral $\mathrm{pH}$ : a review. Appl Catal B Environ 209:358-371. https://doi. org/10.1016/j.apcatb.2017.03.011

Costa RCC, Mourab FCC, Ardisson JD, Fabrisa JD, Lago RM (2008) Highly active heterogeneous Fenton-like systems based on $\mathrm{Fe}_{0} / \mathrm{Fe}_{3} \mathrm{O}_{4}$ composites prepared by controlled reduction of iron oxides. Appl Catal B Environ 83:131-139. https://doi. org/10.1016/j.apcatb.2008.01.039

Cristovao RO, Goncalves C, Botelho CM, Martins RJE (2014) Chemical oxidation of fi canning waste water by Fenton's reagent. J Environ Chem Eng 2:237-243. https://doi.org/10.1016/j. jece.2013.12.023

Czapski G, Bielski BHJ (1993) Absorption spectra of the.OH and O-radicals in aqueous solutions. Radiat Phys Chem 41(3):505. https ://doi.org/10.1016/0969-806X(93)90012-J

Dehghani S, Jafari AJ, Farzadkia M, Gholami M (2013) Sulfonamide antibiotics reduction in aquatic environment by application of Fenton oxidation process. J Environ Health Sci Eng 10:29. https ://doi.org/10.1186/1735-2746-10-29

Descorme C (2017) Catalytic wastewater treatment: oxidation and reduction processes. Recent studies on chlorophenols. Catal Today 297:324-334. https://doi.org/10.1016/j.catto d.2017.03.039

Dhakshinamoorthy A, Navalon S, Alvaro M, Garcia H (2012) Metal nanoparticles as heterogeneous Fenton catalysts. Chem Sus Chem 5(1):46-64. https://doi.org/10.1002/cssc.201100517

Diao Z, Xu X, Jiang D, Li G, Liu J, Kong L, Zuo L (2017) Enhanced catalytic degradation of ciprofloxacin with $\mathrm{FeS}_{2} / \mathrm{SiO}_{2}$ microspheres as heterogeneous Fenton catalyst: kinetics, reaction pathways and mechanism. J Hazard Mater 327:108-115. https ://doi.org/10.1016/j.jhazmat.2016.12.045

Diao Y, Yan Z, Guo M, Wang X (2018) Magnetic multi-metal codoped magnesium ferrite nanoparticles: an efficient visible lightassisted heterogeneous Fenton-like catalyst synthesized from saprolite laterite ore. J Hazard Mater 344:829-838. https://doi. org/10.1016/j.jhazmat.2017.11.029

Dias IN, Souza BS, Pereira JHOS, Moreira FC, Dezotti M, Boaventura RAR, Vilar VJP (2014) Enhancement of the photo-Fenton reaction at near neutral $\mathrm{pH}$ through the use of ferrioxalate complexes: a case study on trimethoprim and sulfamethoxazole antibiotics removal from aqueous solutions. Chem Eng J 247:302-313. https ://doi.org/10.1016/j.cej.2014.03.020

Dindarsafa M, Khataee A, Kaymak B, Vahid B, Karimi A, Rahmani A (2017) Heterogeneous sono-Fenton-like process using martite nanocatalyst prepared by high energy planetary ball milling for treatment of a textile dye. Ultrason Sonochem 34:389-399. https ://doi.org/10.1016/j.ultsonch.2016.06.016

Djowe AT, Nzali S, Njoyim ET, Laminsi S, Gaigneaux EM (2014) Thermal treatment of plasma-synthesized goethite improves Fenton-like degradation of orange II dye. Environ Chem Lett 12:219-224. https://doi.org/10.1007/s10311-016-0578-y

Du D, Shi W, Wang L, Zhang J (2017) Yolk shell structured $\mathrm{Fe}_{3} \mathrm{O}_{4} @$ void@ $\mathrm{TiO}_{2}$ as photo-Fenton-like catalyst for the extremely efficient elimination of tetracycline. Appl Catal B Environ 200:484 492. https://doi.org/10.1016/j.apcatb.2016.07.043

Duan X, Sun H, Shao Z, Wang S (2018) Nonradical reactions in environmental remediation processes: uncertainty and challenges. Appl Catal B Environ 224:973-982. https://doi.org/10.1016/j. apcatb.2017.11.051 
Duarte F, Maldonado-Hodar FJ, Perez-Cadenas AF, Madeira LM (2009) Fenton like degradation of azo dye orange II catalysed by transition metals on carbon aerogels. Appl Catal B Environ 85:139-147. https://doi.org/10.1016/j.apcatb.2008.07.006

Durán A, Monteagudo JM, Sanmartín I, Carrasco A (2013) Solar photo-Fenton mineralization of antipyrine in aqueous solution. J Environ Manag 130:64-71. https://doi.org/10.1016/j.jenvm an.2013.08.043

Elmolla ES, Chaudhuri M (2009a) Optimization of Fenton process for the treatment of amoxicillin, ampicillin, cloxacillin antibiotics in aqueous solution. J Hazard Mater 170:666-672. https://doi. org/10.1016/j.jhazmat.2009.05.013

Elmolla ES, Chaudhuri M (2009b) Degradation of the antibiotics amoxicillin, ampicillin, cloxacillin in aqueous solution by the photo-Fenton process. J Hazard Mater 172:1476-1481. https:// doi.org/10.1016/j.jhazmat.2009.08.015

Elmolla ES, Chaudhuri M (2010a) Comparisons of different advanced oxidation processes for the treatment of antibiotic aqueous solution. Desalination 256:43-47. https://doi.org/10.1016/j.desal .2010 .02 .019

Elmolla ES, Chaudhuri M (2010b) Degradation of amoxicillin, ampicillin, cloxacillin antibiotics in aqueous solution by the UV/ZnO photocatalytic process. J Hazard Mater 173:445-449. https://doi. org/10.1016/j.jhazmat.2009.08.104

Elmolla ES, Chaudhuri M (2012) The feasibility of using combined Fenton SBR for antibiotic wastewater treatment. Desalination 285:14-21. https://doi.org/10.1016/j.desal.2011.09.022

Elmolla ES, Chaudhari M, Eltoukhy MM (2010) The use of artificial neural network (ANN) for modeling of COD removal from antibiotic aqueous solution by the Fenton process. J Hazard Mater 179:127-134. https://doi.org/10.1016/j.jhazmat.2010.02.068

Eren Z (2012) Ultrasound as a basic and auxiliary process for dye remediation: a review. J Environ Manag 104:127-141. https:// doi.org/10.1016/j.jenvman.2012.03.028

Espinosa JC, Navalón S, Álvaro M, García H (2016) Reduced graphene oxide as a metal-free catalyst for the light-assisted Fenton-like reaction. Chem Cat Chem 8(16):2642-2648. https://doi. org/10.1002/cctc. 201600364

Espinosa JC, Catalá C, Navalón S, Ferrer B, Álvaro M, García H (2018) Iron oxide nanoparticles supported on diamond nanoparticles as efficient and stable catalyst for the visible light assisted Fenton reaction. Appl Catal B Environ 226:242-251. https://doi. org/10.1016/j.apcatb.2017.12.060

Espinoza C, Romero J, Villegas L, Cornejo-Ponce L, Salazar R (2016) Mineralization of the textile dye acid yellow 42 by solar photoelectro-Fenton in a lab-pilot plant. J Hazard Mater 319:24-33. https://doi.org/10.1016/j.jhazmat.2016.03.003

Estrada AL, Li YY, Wang A (2012) Biodegradability enhancement of wastewater containing cefalexin by means of the electro Fenton oxidation process. J Hazard Mater 227-228:41-48. https://doi. org/10.1016/j.jhazmat.2012.04.079

Exposito AJ, Monteagudo JM, Diaz I, Duran A (2016) Photo-Fenton degradation of a beverage industrial effluent: intensification with persulfate and the study of radicals. Chem Eng J 306:1203-1211. https://doi.org/10.1016/j.cej.2016.08.048

Fan X, Hao H, Shen X, Chen F, Zhang J (2011) Removal and degradation pathway study of sulfasalazine with Fenton like reaction. J Hazard Mater 190:493-500. https://doi.org/10.1016/j.jhazm at.2011.03.069

Faust BC, Hoigné J (1990) Photolysis of Fe(III)-hydroxy complexes as sources of $\mathrm{OH}$ radicals in clouds, fog and rain. Atmos Environ Part A Gen Top 24(1):79-89. https://doi.org/10.1016/09601686(90)90443-Q

Faust BC, Zepp RG (1993) Photochemistry of aqueous Iron(III)-polycarboxylate complexes: roles in the chemistry of atmospheric and surface waters. Environ Sci Technol 27(12):2517-2527. https:// doi.org/10.1021/es00048a032

Feng J, Hu X, Yue PL (2006) Effect of initial solution pH on the degradation of orange II using clay-based Fe nanocomposites as heterogeneous photo Fenton catalyst. Water Res 40:641-646. https ://doi.org/10.1016/j.watres.2005.12.021

Feng L, Van Hullebusch ED, Rodrigo MA, Esposito G, Oturan MA (2013) Removal of residual anti-infl ory and analgesic pharmaceuticals from aqueous systems by electrochemical advanced oxidation processes: a review. Chem Eng J 228:944964. https://doi.org/10.1016/j.cej.2013.05.061

Feng M, Wang X, Chen J, Qu R, Sui Y, Cizmas L, Wang Z, Sharma VK (2016) Degradation of fluoroquinolone antibiotics by ferrate (VI): eff of water constituent and oxidized products. Water Res 103:48-57. https://doi.org/10.1016/j.watres.2016.07.014

Feng M, Wang Z, Dionysiou DD, Sharma VK (2018) Metal-mediated oxidation of $\mathrm{fl}$ oquinolone antibiotics in water: a review on kinetics, transformation products, and toxicity assessment. J Hazard Mater 344:1136-1154. https://doi.org/10.1016/j.jhazm at.2017.08.067

Fenton HJH (1894) LXXIII.- oxidation of tartaric acid in presence of iron. J Chem Soc Trans 65:899. https://doi.org/10.1039/ct894 6500899

Fenton HJH (1896) XLI.- the constitution of a new dibasic acid, resulting from the oxidation of tartaric acid. J Chem Soc Trans 69:575. https://doi.org/10.1039/ct8966900546

Fischbacher A, Von Sonntag C, Schmidt TC (2017) Hydroxyl radical yields in the Fenton process under various $\mathrm{pH}$, ligand concentrations and hydrogen peroxide/Fe(II) ratios. Chemosphere 182:738-744. https://doi.org/10.1016/j.chemospher e.2017.05.039

Gallard H, De Laat J (2000) Kinetic modelling of $\mathrm{Fe}(\mathrm{III}) / \mathrm{H}_{2} \mathrm{O}_{2}$ oxidation reactions in dilute aqueous solution using atrazine as a model organic compound. Water Res 34(12):3107-3116. https ://doi.org/10.1016/S0043-1354(00)00074-9

Gallard H, De Laat J, Legube B (1999) Spectrophotometric study of the formation of iron(III)-hydroperoxy complexes in homogeneous aqueous solutions. Water Res 33(13):2929-2936. https://doi. org/10.1016/S0043-1354(99)00007-X

Gan PP, Li SFY (2013) Effi removal of rhodamine B using a rice hull-based silica supported iron catalyst by Fenton like process. Chem Eng J 229:351-363. https://doi.org/10.1016/j. cej.2013.06.020

Ganzenko O, Trellu C, Papirio S, Oturan N, Huguenot D, van Hullebusch ED, Esposito G, Oturan MA (2017) Bioelectro-Fenton: evaluation of a combined biological-advanced oxidation treatment for pharmaceutical wastewater. Environ Sci Pollut Res. https://doi.org/10.1007/s11356-017-8450-6

García-Rodríguez O, Bañuelos JA, Godínez LA, Arredondo Valdez HC, Zamudio E, Ramírez V, Rodríguez-Valadez FJ (2017) Iron Supported on Ion Exchange Resin as Source of Iron for Fenton's reagent: a Heterogeneous or a Homogeneous Fenton's reagent Generation? Int J Chem Reactor Eng. https://doi.org/10.1515/ ijcre-2017-0026

Garcia-Segura S, Brillas E (2017) Applied photoelectrocatalysis on the degradation of organic pollutants in wastewaters. J Photochem Photobiol C Photochem Rev 31:1-35. https://doi.org/10.1016/j. jphotochemrev.2017.01.005

Gassie LW, Englehardt JD (2017) Advanced oxidation and disinfection processes for onsite net-zero greywater reuse: a review. Water Res 125:384-399. https://doi.org/10.1016/j.watres.2017.08.062

Ghattas A, Fischer F, Wick A, Ternes TA (2017) Anaerobic biodegradation of (emerging) organic contaminants in the aquatic environment. Water Res 116:268-295. https://doi.org/10.1016/j.watre s.2017.02.001 
Ghenymy AE, Rodriguez RM, Email EB, Oturan N, Oturan MA (2014) Electro-Fenton degradation of the antibiotic sulfanilamide with $\mathrm{Pt} /$ carbon-felt and BDD/carbon-felt cells. Kinetics, reaction intermediates and toxicity assessment. Environ Sci Pollut Res 2(14):8368-8378. https://doi.org/10.1007/s1135 6-014-2773-3

Ghosh P, Samanta AN, Ray S (2011) Kinetics based on mechanism of COD reduction for industrial effl in Fenton process. Int J Chem Technol 3(1):26-36. https://doi.org/10.3923/ ijct.2011.26.36

Giraldo-Aguirre AL, Serna-Galvis A, Erazo-Erazo ED, Agredo JS, Ospina HG, Acosta OAF, Palma AT (2017) Removal of $\beta$-lactam antibiotics from pharmaceutical wastewaters using photo-Fenton process at near neutral pH. Environ Sci Pollut Res. https://doi. org/10.1007/s11356-017-8420-z

Giri AS, Golder AK (2015) Decomposition of drug mixture in Fenton and photo Fenton processes: comparison to singly treatment, evolution of inorganic ions and toxicity assay. Chemosphere 127:254-261. https://doi.org/10.1016/j.chemospher e.2015.02.010

Gligorovski S, Strekowski R, Barbati S, Vione D (2015) Environmental implications of hydroxyl radicals $(\mathrm{OH})$. Chem Rev 115(24):13051-13092. https://doi.org/10.1021/cr500310b

Golash N, Gogate PR (2012) Degradation of dichlorvos containing wastewaters using sonochemical reactor. Ultrason Sonochem 19:1051-1060. https://doi.org/10.1016/j.ultsonch.2012.02.011

Goldstein S, Meyersteion D (1999) Comments on the mechanism of the "Fenton-like" reaction. Acc Chem Res 32:547-555. https:// doi.org/10.1021/ar9800789

Gong Y, Li J, Zhang Y, Zhang M, Tian X, Wang A (2016) Partial degradation of levofl xacin for biodegradability improvement by electro Fenton process using an activated carbon fi felt cathode. J Hazard Mater 304:320-328. https://doi.org/10.1016/j. jhazmat.2015.10.064

Gonzalez O, Sans C, Esplugas S (2007) Sulfamethoxazole abatement by photo-Fenton toxicity, inhibition and biodegradability assessment of intermediates. J Hazard Mater 146:459-464. https://doi. org/10.1016/j.jhazmat.2007.04.055

Gozzi F, Sirés I, Thiam A, de Oliveira SC, Junior AM, Brillas E (2017) Treatment of single and mixed pesticide formulations by solar photoelectro-Fenton using a flow plant. Chem Eng J 310:503513. https://doi.org/10.1016/j.cej.2016.02.026

Gu L, Zhu N, Guo H, Huang S, Lou Z, Yuan H (2013) Adsorption and Fenton like degradation of naphthalene dye intermediate on sewage sludge derived porous carbon. J Hazard Mater 246-247:145153. https://doi.org/10.1016/j.jhazmat.2012.12.012

Gu S, Xieb J, Li CM (2014) Hierarchically porous graphitic carbon nitride: large scale facile synthesis and its application toward photocatalytic dye degradation. RSC Adv 4:59436-59439. https ://doi.org/10.1039/C4RA10958A

Gulkowska A, Leunga HW, Soa MK, Taniyasub S, Yamashit N, Yeung LWY, Richardson BJ, Lei AP, Giesy JP, Lam PKS (2008) Removal of antibiotics from wastewater by sewage treatment facilities in Hong Kong and Shenzhen, China. Water Res 42:395-403. https://doi.org/10.1016/j.watres.2007.07.031

Hajj-Mohamad M, Darwano H, Duy SV, Sauvé S, Prévost M, Arp HPH, Dorner S (2017) The distribution dynamics and desorption behaviour of mobile pharmaceuticals and caffeine to combined sewer sediments. Water Res 108:57-67. https://doi.org/10.1016/j. watres.2016.10.053

He H, Zhou Z (2017) Electro-Fenton process for water and wastewater treatment. Crit Rev Environ Sci Technol 47:2100-2131. https:// doi.org/10.1080/10643389.2017.1405673

Hirsch R, Ternes TA, Haberer K, Kratz KL (1999) Occurrence of antibiotics in the aquatic environment. Sci Total Environ 225:109118. https://doi.org/10.1016/S0048-9697(98)00337-4
Homem V, Alves A, Santos L (2010) Amoxicillin degradation at ppb levels by Fenton's oxidation using design of experiments. Sci Total Environ 408:6272-6280. https://doi.org/10.1016/j.scito tenv.2010.08.058

Hou L, Wang L, Royer S, Zhang H (2016) Ultrasound assisted heterogeneous Fenton like degradation of tetracycline over a magnetite catalyst. J Hazard Mater 302:458-467. https://doi.org/10.1016/j. jhazmat.2015.09.033

Hsu CA, Wen TN, Su YC, Ziang ZB, Chen CW, Shyur LF (2012) Biological degradation of anthraquinone and azo dyes by a novel laccase from lentinus sp. Environ Sci Technol 46(9):5109-5117. https://doi.org/10.1021/es2047014

Huang C, Huang Y, Cheng H, Huang Y (2009) Kinetic study of an immobilized iron oxide for catalytic degradation of azo dye reactive black B with catalytic decomposition of hydrogen peroxide. Catal Commun 10(5):561-566. https://doi.org/10.1016/j.catco m.2008.10.033

Hug SJ, Leupin O (2003) Iron-catalyzed oxidation of Arsenic(III) by oxygen and by hydrogen peroxide: $\mathrm{pH}$-dependent formation of oxidants in the Fenton reaction. Environ Sci Technol 37(12):2734-2742. https://doi.org/10.1021/es026208x

Hui SJ, Lan FJ, Hui SS, Qing PIY, Ke SM, Yan S (2012) Degradation of the antibiotic sulfamonomethoxine sodium in aqueous solution by photo-Fenton oxidation. Environ Sci Technol 57:558-564. https://doi.org/10.1007/s11434-011-4887-z

Isarain-Chávez E, Garrido JA, Rodríguez RM, Centellas F, Arias C, Cabot PL, Brillas E (2011) Mineralization of metoprolol by electro-Fenton and photoelectro-Fenton processes. J Phys Chem A 115(7):1234-1242. https://doi.org/10.1021/jp110753r

Jafari N, Kasra-Kermanshashi R, Soud MR, Mahvi AH, Gharavi S (2012) Degradation of a textile reactive azo dye by a combine biological-photocatalyticprocess: candidatropicalis $\mathrm{Jks}_{2}-\mathrm{TiO}_{2} /$ UV. Iran J Environ Health Sci Eng 9(33):1-7. https://doi. org/10.1186/1735-2746-9-33

Kakavandi B, Takdastan A, Jafaarzadeh N, Azizi M, Mirzaei A, Azari A (2016) Application of $\mathrm{Fe}_{3} \mathrm{O}_{4} @ \mathrm{C}$ catalyzing heterogeneous UV-Fenton system for tetracycline removal with a focus on optimization by a response surface method. J Photochem Photobiol A Chem 314:178-188. https://doi.org/10.1016/j.jphotochem 2015.08.008

Kalishwaralal K, Jeyabharathi S, Sundar K, Muthukumaran A (2016) A novel one-pot green synthesis of selenium nanoparticles and evaluation of its toxicity in zebrafi embryos. Artif Cells Nanomed Biotechnol 44(2):471-477. https://doi. org/10.3109/21691401.2014.962744

Kang S, Bokare AD, Park Y, Choi CH, Choi W (2017) Electron shuttling catalytic effect of mellitic acid in zero-valent iron induced oxidative degradation. Catal Today 282:65-70. https://doi. org/10.1016/j.cattod.2016.03.009

Karaolia P, Michael I, Fernandez IC, Aguera A, Malato S, Ibanez PF, Kassinos DF (2014) Reduction of clarithromycin and sulfamethoxazole resistant enterococcus by pilot scale solar driven Fenton oxidation. Sci Total Environ 468-469:19-27. https://doi. org/10.1016/j.scitotenv.2013.08.027

Karaolia P, Koradatou IM, Hapeshi E, Schwartz JT, Kassinos DF (2017) Investigation of the potential of membrane bioreactor followed by solar Fenton oxidation to remove antibiotic related microcontaminants. Chem Eng J 310:491-502. https://doi. org/10.1016/j.cej.2016.04.113

Kasiri MB, Aleboyeh H, Aleboyeh A (2008) Degradation of acid blue 74 using Fe-ZSM5 zeolite as a heterogeneous photo-Fenton catalyst. Appl Catal B Environ 84(1-2):9-15. https://doi. org/10.1016/j.apcatb.2008.02.024

Kassinos DF, Vasquez MI, Kummerer K (2011) Transformation products of pharmaceuticals in surface waters and waste water formed during photolysis and advanced oxidation processes: 
degradation, elucidation of byproducts and assessment of their biological potency. Chemosphere 85(5):693-709. https://doi. org/10.1016/j.chemosphere.2011.06.082

Katsoyiannis IA, Ruettimann T, Hug SJ (2008) pH dependence of Fenton reagent generation and As(III) oxidation and removal by corrosion of zero valent iron in aerated water. Environ Sci Technol 42(19):7424-7430. https://doi.org/10.1021/es800649p

Kaur R, Wani SP, Singh SP, Singh AK, Lal K (2012) Waste water production, treatment and use in India

Khataee A, Gholami P, Vahid B, Joo SW (2016) Heterogenous sono-Fenton process using pyrite nanorods prepared by nonthermal plasma for degradation of an anthraquinone dye. Ultrason Sonochem 32:357-370. https://doi.org/10.1016/j.ultso nch.2016.04.002

Kim H, Hwang YS, Sharma VK (2014) Adsorption of antibiotics and iopromide onto single-walled and multi-walled carbon nanotubes. Chem Eng J 255:23-27. https://doi.org/10.1016/j. cej.2014.06.035

Kitsiou V, Antoniadis A, Mantzavinos D, Poulios I (2014) Homogeneous photo-Fenton mineralization of the antibiotic sulfamethazine in water under UV-A visible and solar irradiation. J Chem Technol Biotechnol 89:1668-1674. https://doi.org/10.1002/jctb.4237

Klatte S, Schaefer H, Hempel M (2017) Pharmaceuticals in the environment-a short review on options to minimize the exposure of humans, animals and ecosystems. Sustain Chem Pharm 5:61-66. https://doi.org/10.1016/j.scp.2016.07.001

Kralchevska RP, Prucek R, Kolarík J, Tucek J, Machala L, Filip J, Sharma VK, Zboril R (2016) Remarkable effi of phosphate removal: Ferrate(VI)-induced in situ sorption on core-shell nanoparticles. Water Res 103:83-91. https://doi.org/10.1016/j. watres.2016.07.021

Le C, Liang J, Wu J, Li P, Wang X, Zhu N, Wu P, Yang B (2011) Effective degradation of para-chloronitrobenzene through a sequential treatment using zero-valent iron reduction and Fenton oxidation. Water Sci Technol 64(10):2126-2131. https://doi.org/10.2166/ wst.2011.803

Li Y, Lu Y, Zhu X (2006) Photo-Fenton discoloration of the azo dye $\mathrm{X}-3 \mathrm{~B}$ over pillared bentonites containing iron. J Hazard Mater 132:196-201. https://doi.org/10.1016/j.jhazmat.2005.07.090

Li J, Mailhot G, Wu F, Deng N (2012) Photodegradation of E2 in the presence of natural montmorillonite and the iron complexing agent ethylenediamine-N,N'-disuccinic acid. Photochem Photobiol Sci. https://doi.org/10.1039/c2pp25159k

Li H, Pan Y, Wang Z, Chen S, Guo R, Chen J (2015) An algal process treatment combined with the Fenton reaction for high concentrations of amoxicillin and cefradine. RSC Adv 5:100775-100782. https://doi.org/10.1039/C5RA21508K

Li L, Song C, Huang Y, Zhou Y (2016) Investigation of BTEX removal efficiency using the electrolytic oxidation and Fenton's reaction. J Water Chem Technol 38:149-157. https://doi.org/10.3103/S1063 455X1603005X

Li X, Zhu K, Pang J, Tian M, Liu J, Rykov AI, Zheng M, Wang X, Zhu X, Huang Y, Liu B, Wang J, Yang W, Zhang T (2018) Unique role of Mossbauer spectroscopy in assessing structural features of heterogeneous catalysts. Appl Catal B Environ 224:518-532. https://doi.org/10.1016/j.apcatb.2017.11.004

Lima MJ, Leblebici ME, Dias MM, Lopes JCB, Silva CG, Silva AMT, Faria JL (2014) Continuous flow photo-Fenton treatment of ciprofl xacin in aqueous solution using homogeneous and magnetically recoverable catalyst. Environ Sci Pollut Res 21:111611125. https://doi.org/10.1007/s11356-014-2515-6

Lima MJ, Silva CG, Silva AMT, Lopes JCB, Dias MM, Faria JL (2017) Homogeneous and heterogeneous photo-Fenton degradation of antibiotics using an innovative static mixer photoreactor. Chem Eng J 310:342-351. https://doi.org/10.1016/j.cej.2016.04.032
Lin H, Oturan N, Wu J, Sharma VK, Zhang H, Oturan MA (2017a) Removal of artificial sweetener aspartame from aqueous media by electrochemical advanced oxidation processes. Chemosphere 167:220-227. https://doi.org/10.1016/j.chemospher e.2016.09.143

Lin H, Oturan N, Wu J, Zhang H, Oturan MA (2017b) Cold incineration of sucralose in aqueous solution by electro-Fenton process. Sep Purif Technol 173:218-225. https://doi.org/10.1016/j.seppu r.2016.09.028

Liu J, Yang W (2012a) Water sustainability for China and beyond. Science 337:649-650. https://doi.org/10.1126/science.1219471

Liu J, Yang W (2012b) Water sustainability for China and beyond. Science. https://doi.org/10.1126/science.1219471

Liu C, Wu B, ChLiu C, Wu B, Chen X (2018) Sulfate radical-based oxidation for sludge treatment: A review. Chem Eng J. https:// doi.org/10.1016/j.cej.2017.10.162

Loannou L, Velegraki T, Michael C, Mantzavinos D, Kassinos DF (2013) Sunlight, iron and radicals to tackle the resistant leftovers of bio treated winery wastewater. Photochem Photobiol Sci 12:664-670. https://doi.org/10.1039/C2PP25192B

Lucas MS, Dias AA, Sampaio A, Amaral C, Peres J (2007) Degradation of a textile reactive Azo dye by a combined chemical-biological process: Fenton's reagent-yeast. Water Res 41(5):1103-1109. https://doi.org/10.1016/j.watres.2006.12.013

Lyu L, Hu C (2017) Heterogeneous Fenton catalytic water treatment technology and mechanism. Progr Chem 29:981-999. https://doi. org/10.7536/PC170552

Ma Y (2012) Short review: current trends and future challenges in the application of sono-Fenton oxidation for wastewater treatment. Sustain Environ Res 22(5):271-278

Ma YS, Sung CF, Lin JG (2010) Degradation of carbofuran in aqueous solution by ultrasound and Fenton process: effect of system parameters and kinetic study. J Hazard Mater 178:320-325. https ://doi.org/10.1016/j.jhazmat.2010.01.081

Mandal T, Maity S, Dasgupta D, Datta S (2010) Advanced oxidation process and biotreatment: their roles in combined industrial wastewater treatment. Desalination 250(1):87-94. https://doi. org/10.1016/j.desal.2009.04.012

Matafonova G, Batoev V (2018) Recent advances in application of UV light-emitting diodes for degrading organic pollutants in water through advanced oxidation processes: a review. Water Res 132:177-189. https://doi.org/10.1016/j.watres.2017.12.079

Michael I, Hapeshi E, Michael C, Varela AR, Kyriakou S, Manaia CM, Kassinos DF (2012) Solar photo-Fenton process on the abatement of antibiotics at a pilot scale: degradation kinetics, ecotoxicity and phytotoxicity assessment and removal of antibiotic resistant enterococci. Water Res 46:5621-5634. https://doi. org/10.1016/j.watres.2012.07.049

Michael I, Hapeshi E, Acena J, Perez S, Petrovic M, Zapata A, Barcelo D, Malato S, Kassinos DF (2013) Light-induced catalytic transformation of ofloxacin by solar Fenton in various water matrices at a pilot plant: mineralization and characterization of major intermediate products. Sci Total Environ 461-462:39-48. https ://doi.org/10.1016/j.scitotenv.2013.04.054

Mirzaei A, Chen Z, Haghighat F, Yerushalmi L (2017) Removal of pharmaceuticals from water by homo/heterogonous Fenton-type processes-a review. Chemosphere 174:665-688. https://doi. org/10.1016/j.chemosphere.2017.02.019

Mohammadi AS, Yazdanbakhsh AR, Sardar M (2013) Chemical oxygen demand removal from synthetic wastewater containing non-beta lactam antibiotics using advanced oxidation process: a comparative study. Arch Hyg Sci 2(1):23-30. http://jhygiene. muq.ac.ir/article-1-122-en.html

Morales-Pérez AA, Arias C, Ramírez Zamora R (2016a) Removal of atrazine from water using an iron photo catalyst supported on 
activated carbon. Adsorpt 22:48-58. https://doi.org/10.1007/ s10450-015-9739-8

Morales-Pérez AA, Maravilla P, Solís-López M, Schouwenaars R, Durán-Moreno A, Ramírez-Zamora R (2016b) Optimization of the synthesis process of an iron oxide nanocatalyst supported on activated carbon for the inactivation of Ascaris eggs in water using the heterogeneous Fenton-like reaction. Water Sci Technol 73(5):1000-1009. https://doi.org/10.2166/wst.2015.576

Moreira FC, Soler J, Alpendurada MF, Boaventura RAR, Brillas E, Vilar VJP (2016) Tertiary treatment of a municipal wastewater toward pharmaceuticals removal by chemical and electrochemical advanced oxidation processes. Water Res 105:251-263. https ://doi.org/10.1016/j.watres.2016.08.036

Mousset E, Frunzo L, Esposito G, Van Hullebusch ED, Oturan N, Oturan MA (2016) A complete phenol oxidation pathway obtained during electro-Fenton treatment and validated by a kinetic model study. Appl Catal B Environ 180:189-198. https ://doi.org/10.1016/j.apcatb.2015.06.014

Mousset E, Oturan N, Oturan MA (2018) An unprecedented route of $\mathrm{OH}$ radical reactivity evidenced by an electrocatalytical process: ipso-substitution with per halogeno carbon compounds. Appl Catal B Environ 226:135-146. https://doi.org/10.1016/j.apcat b.2017.12.028

Moya MP, Graells M, Castells G, Amigo J, Ortega E, Buhigas G, Perez LM, Mansilla HD (2010) Characterization of the degradation performance of the sulfamethazine antibiotic by photo-Fenton process. Water Res 44:2533-2540. https://doi.org/10.1016/j. watres.2010.01.032

Nidheesh PV, Olvera-Vargas H, Oturan N, Oturan MA (2017) Heterogeneous electro-Fenton process: principles and applications. In: Zhou M, Oturan MA, Sirés I (eds) Electro-Fenton process: new trends and scale-up. Handbook of Environmental Chemistrt, vol 61. Springer, Singapore, pp 85-110. https://doi. org/10.1007/698_2017_72

Nidheesh PV, Zhou M, Oturan MA (2018) An overview on the removal of synthetic dyes from water by electrochemical advanced oxidation processes. Chemosphere 197:210-227. https://doi. org/10.1016/j.chemosphere.2017.12.195

Olvera-Vargas H, Cocerva T, Oturan N, Buisson D, Oturan MA (2016a) Bioelectro-Fenton: a sustainable integrated process for removal of organic pollutants from water: application to mineralization of metoprolol. J Hazard Mater 319:13-23. https://doi.org/10.1016/j. jhazmat.2015.12.010

Olvera-Vargas H, Oturan N, Buisson D, Oturan MA (2016b) A coupled Bio-EF process for mineralization of the pharmaceuticals Furosemide and Ranitidine: feasibility assessment. Chemosphere 155:606-613. https://doi.org/10.1016/j.chemospher e.2016.04.091

Oturan MA, Aaron J-J (2014) Advanced oxidation processes in water/ wastewater treatment: principles and applications: a review. Crit Rev Environ Sci Technol 44:2577-2641. https://doi. org/10.1080/10643389.2013.829765

Oturan E, Oturan N, Oturan MA (2018) An unprecedented route of [rad]OH radical reactivity evidenced by an electrocatalytical process: Ipso-substitution with perhalogenocarbon compounds. Appl Catal B Environ. https://doi.org/10.1016/j.apcatb.2017.12.028

Oturan MA, Sirés I, Oturan N, Pérocheau S, Laborde J, Trévin S (2008) Sonoelectro-Fenton process: a novel hybrid technique for the destruction of organic pollutants in water. J Electroanal Chem 624(1-2):329-332. https://doi.org/10.1016/j.jelec hem.2008.08.005

Oturan N, Wu J, Zhang H, Sharma VK, Oturan MA (2013) Electrocatalytic destruction of the antibiotic tetracycline in aqueous medium by electrochemical advanced oxidation processes: effect of electrode materials. Appl Catal B Environ 140-141:92-97. https:// doi.org/10.1016/j.apcatb.2013.03.035
Ouiriemmi I, Karrab A, Oturan N, Pazos M, Rozales E, Gadri A, Sanromán MÁ, Ammar S, Oturan MA (2017) Heterogeneous electro-Fenton using natural pyrite as solid catalyst for oxidative degradation of vanillic acid. J Electroanal Chem 797:6977. https://doi.org/10.1016/j.jelechem.2017.05.028

Özcan A, Atilir Özcan A, Demirci Y (2016) Evaluation of mineralization kinetics and pathway of norfl xacin removal from water by electro-Fenton treatment. Chem Eng J 304:518-526. https ://doi.org/10.1016/j.cej.2016.06.105

Özdemir C, Öden MK, Sahinkaya S, Kalipçi E (2011) Color removal from synthetic textile wastewater by sono-Fenton process. CLEAN Soil Air Water 39(1):60-67. https://doi.org/10.1002/ clen.201000263

Pi L, Yang N, Han W, Xiao W, Wang D, Xiong Y, Zhou M, Hou H, Mao X (2018) Heterogeneous activation of peroxymonocarbonate by Co-Mn oxides for the efficient degradation of chlorophenols in the presence of a naturally occurring level of bicarbonate. Chem Eng J 334:1297-1308. https://doi.org/10.1016/j. cej.2017.11.006

Pignatello JJ, Oliveros E, MacKay A (2006) Advanced oxidation processes for organic contaminant destruction based on the Fenton reaction and related chemistry. Crit Rev Environ Sci Technol 36:1-84. https://doi.org/10.1080/10643380500326564

Pliego G, Zazo JA, Garcia-Muñoz P, Munoz M, Casas JA, Rodriguez JJ (2015) Trends in the intensification of the Fenton process for wastewater treatment: an overview. Crit Rev Environ Sci Technol 36:1-84. https://doi.org/10.1080/10643389.2015.1025646

Poerscchmann J, Trommler U, Gorecki T (2010) Aromatic intermediate formation during oxidative degradation of bisphenol A by homogeneous sub stoichiometric Fenton reaction. Chemosphere 79:975-986. https://doi.org/10.1016/j.chemospher e.2010.03.030

Prachi K, Anushree M (2009) Fungal dye decolourization: recent advances and future potential. Environ Int 35:127-141. https ://doi.org/10.1016/j.envint.2008.05.010

Pulicharla R, Brar SK, Rouissi T, Auger S, Drogui P, Verma M, Surampalli RY (2017) Degradation of chlortetracycline in waste water sludge by ultrasonication, Fenton oxidation and ferro sonication. UltrasonSonochem 34:332-342. https://doi. org/10.1016/j.ultsonch.2016.05.042

Rachmilovich-Calis S, Masarwa A, Meyerstein N, Meyerstein DvE R (2009) New mechanistic aspects of the Fenton reaction. Chem Eur J 15:8303-8309. https://doi.org/10.1002/chem.200802572

Ranjit PJD, Palanivelu K, Lee CS (2008) Degradation of 2,4-dichlorophenol in aqueous solution by sono-Fenton method. Korean J Chem Eng 25:112-117. https://doi.org/10.1007/s1181 4-008-0020-7

Rozas O, Contreras D, Mondaca MA, Moya MP, Mansilla HD (2010) Experimental design of Fenton and photo Fenton reactions for the treatment of ampicillin solutions. J Hazard Mater 177:1025-1030. https://doi.org/10.1016/j.jhazmat.2010.01.023

Rush JD, Bielski BHJ (1985) Pulse radiolytic studies of the reactions of $\mathrm{HO}_{2} / \mathrm{O}^{2-}$ with $\mathrm{Fe}(\mathrm{II}) / \mathrm{Fe}(\mathrm{III})$ ions. The reactivity of $\mathrm{HO}_{2} / \mathrm{O}_{2}{ }^{-}$ with ferric ions and its implication on the occurrence of the Haber-Weiss reaction. J Phys Chem 89(23):5062-5066. https ://doi.org/10.1021/j100269a035

Salimi M, Esrafi A, Gholami M, Jonidi Jafari A, Rezaei Kalantary R, Farzadkia M, Kermani M, Sobhi HR (2017) Contaminants of emerging concern: a review of new approach in AOP technologies. Environ Monit Assess 189(8):414. https://doi. org/10.1007/s10661-017-6097-x

Sanchez PTA, Hernandez IL, Miranda VM, Lugo VL, De-oca RMGFM (2014) Waste water treatment of methyl methacrylate by Fenton's reagent and adsorption. Catal Today 220-222:3948. https://doi.org/10.1016/j.cattod.2013.09.006 
Santos LVDS, Meireles AM, Lange LC (2015) Degradation of antibiotics norfloxacin by Fenton, $\mathrm{UV}$ and $\mathrm{UV} / \mathrm{H}_{2} \mathrm{O}_{2}$. J Environ Manag 154:8-12. https://doi.org/10.1016/j.jenvman.2015.02.021

Sarria V, Parra S, Invernizzi M, Pulgarin C (2001) Photochemicalbiological treatment of a real industrial biorecalcitrant waste water containing 5-amino-6-methyl-2-benzimidazolone. Water Sci Technol 44:93-101 PMID:11695489

Schmeller DS, Loyau A, Bao K, Brack W, Chatzinotas A, De Vleeschouwer F, Friesen J, Gandois L, Hansson SV, Haver M, Le Roux G, Shen J, Teisserenc R, Vredenburg VT (2018) People, pollution and pathogens-global change impacts in mountain freshwater ecosystems. Sci Total Environ 622-623:756-763. https://doi.org/10.1016/j.scitotenv.2017.12.006

Schrank SG, Jose HJ, Moreira RFPM, Schroder HF (2005) Applicability of Fenton and $\mathrm{H}_{2} \mathrm{O}_{2} / \mathrm{UV}$ reaction in the treatment of tannery waste waters. Chemosphere 60:644-655. https://doi. org/10.1016/j.chemosphere.2005.01.033

Schwarzenbach RP, Escher BI, Fenner K, Hofstetter TB, Johnson CA, Von Gunten U, Wehrli B (2006) The challenge of micropollutants in aquatic systems. Science 313:1072-1077. https://doi. org/10.1126/science.1127291

Schwarzenbach RP, Egli T, Hofstetter TB, Von Gunten U, Wehrli B (2010) Global water pollution and human health. Annu Rev Environ Resour 35:109-136. https://doi.org/10.1146/annurev-envir on-100809-125342

Serpone N, Artemev YM, Ryabchuk VK, Emeline AV, Horikoshi S (2017) Light-driven advanced oxidation processes in the disposal of emerging pharmaceutical contaminants in aqueous media: a brief review. Curr Opin Green Sustain Chem 6:18-33. https:// doi.org/10.1016/j.cogsc.2017.05.003

Shannon MA, Bohn PW, Elimelech M, Georgiadis JG, Mrinas BJ, Mayes AM (2008) Science and technology for water purifi tion in the coming decades. Nature 452:301-310. https://doi. org/10.1038/nature06599

Sharma VK (2013) Oxidation of amino acids, peptides, and proteins. Wiley Inc, New Jersey

Sharma VK, Feng M (2017) Water depollution using metal-organic frameworks-catalyzed advanced oxidation processes: a review. J Hazard Mater. https://doi.org/10.1016/j.jhazmat.2017.09.043

Sharma VK, Sohn M (2009) Aquatic arsenic: toxicity, speciation, transformations, and remediation. Environ Int 35:743-759. https://doi. org/10.1016/j.envint.2009.01.005

Sharma VK, McDonald TJ, Kim H, Garg VK (2015) Magnetic graphene-carbon nanotube iron nanocomposites as adsorbents and antibacterial agents for water purification. Adv Colloid Interface Sci 225:229-240. https://doi.org/10.1016/j.cis.2015.10.006

Sharma VK, Johnson N, Cizmas L, McDonald TJ, Kim H (2016) A review of the infl of treatment strategies on antibiotic resistant bacteria and antibiotic resistance genes. Chemosphere 150:702-714. https://doi.org/10.1016/j.chemospher e. 2015.12 .084

Shimizu A, Tokumura M, Nakajima K, Kawase Y (2012) Phenol removal using zero-valent iron powder in the presence of dissolved oxygen: roles of decomposition by the Fenton reaction and adsorption/precipitation. J Hazard Mater 201-201:60-67. https://doi.org/10.1016/j.jhazmat.2011.11.009

Shriwas AK, Gogate PR (2011) Ultrasonic degradation of methyl parathion in aqueous solutions: intensifi using additives and scale up aspects. Sep Purif Technol 79:1-7. https://doi. org/10.1016/j.seppur.2011.02.034

Sillanpää M, Ncibi MC, Matilainen A (2018) Advanced oxidation processes for the removal of natural organic matter from drinking water sources: a comprehensive review. J Environ Manag 208:56-76. https://doi.org/10.1016/j.jenvman.2017.12.009

Silva CR, Maniero MG, Rath S, Guimaraes JR (2013) Degradation of flumequine by the Fenton and photo Fenton processes: evaluation of residual antimicrobial activity. Sci Total Environ 445446:337-346. https://doi.org/10.1016/j.scitotenv.2012.12.079

Sirés I, Brillas E, Oturan MA, Rodrigo MA, Panizza M (2014) Electrochemical advanced oxidation processes: today and tomorrow: a review. Environ Sci Pollut Res 21(14):8336-8367. https://doi. org/10.1007/s11356-014-2783-1

Sousa JCG, Ribeiro AR, Barbosa MO, Pereira MFR, Silva AMT (2018) A review on environmental monitoring of water organic pollutants identified by EU guidelines. J Hazard Mater 344:146-162. https://doi.org/10.1016/j.jhazmat.2017.09.058

Steter JR, Brillas E, Sirés I (2018) Solar photoelectro-Fenton treatment of a mixture of parabens spiked into secondary treated wastewater effluent at low input current. Appl Catal B Environ 224:410-418. https://doi.org/10.1016/j.apcatb.2017.10.060

Sum OS, Feng J, Hu X, Po L (2004) Pillared laponite clay-based Fe nanocomposites as heterogeneous catalyst for photo Fenton degradation of acid black 1. Chem Eng Sci 59:5269-5275. https:// doi.org/10.1016/j.ces.2004.09.032

Tayo LL, Caparanga AR, Doma BT, Liao C (2018) A Review on the removal of pharmaceutical and personal care products (PPCPs) using advanced oxidation processes. J Adv Oxid Technol 21(1):196-214. https://doi.org/10.26802/jaots.2017.0079

Tekin H, Bilkay O, Ataberk SS, Balta TH, Ceribasi IH, Sanin FD, Dilek FB, Yetis U (2006) Use of Fenton oxidation to improve the biodegradability of a pharmaceutical waste water. J Hazard Mater 136:258-265. https://doi.org/10.1016/j.jhazmat.2005.12.012

Trellu C, Mousset E, Pechaud Y, Huguenot D, van Hullebusch ED, Esposito G, Oturan MA (2016) Removal of hydrophobic organic pollutants from soil washing/flushing solutions: a critical review. J Hazard Mater 306:149-174. https://doi.org/10.1016/j.jhazm at.2015.12.008

Trovo AG, Nogueira RFP, Aguera A, Alba ARF, Sirtori C, Malato S (2009) Degradation of sulfamethoxazole in water by solar Fenton-Chemical and toxicological evaluation. Water Res 43:39223931. https://doi.org/10.1016/j.watres.2009.04.006

Trovo AG, Nogueira RFP, Aguera A, Alba ARF, Malato S (2011) Degradation of the antibiotics amoxicillin by photo-Fenton processChemical and toxicological assessment. Water Res 45(3):13941402. https://doi.org/10.1016/j.watres.2010.10.029

Turbay EY, Jaen E, Graells M, Moya MP (2013) Enhanced photo Fenton process for tetracycline degradation using efficient hydrogen peroxide dosage. J Photochem Photobiol A Chem 267:11-16. https://doi.org/10.1016/j.jphotochem.2013.05.008

Uribe IO, Mosquera-Corral A, Lema JM, Esplugas S (2015) Advanced technologies for water treatment and reuse. AIChE J 61(10):3146-3159. https://doi.org/10.1002/aic.15013

Usman M, Hanna K, Haderlein S (2016) Fenton oxidation to remediate PAHs in contaminated soils: a critical review of major limitations and counter-strategies. Sci Total Environ 569-570:179-190. https://doi.org/10.1016/j.scitotenv.2016.06.135

Velichkova F, Delmas H, Julcour C, Koumanova B (2017) Heterogeneous Fenton and photo-Fenton oxidation for paracetamol removal using iron containing ZSM-5 zeolite as catalyst. AIChE J 63(2):397-854. https://doi.org/10.1002/aic.15369

Vidal J, Huiliñir C, Santander R, Silva-Agredo J, Torres-Palma RA, Salazar R (2018) Effective removal of the antibiotic nafcillin from water by combining the photoelectro-Fenton process and anaerobic biological digestion. Sci Total Environ 624:10951105. https://doi.org/10.1016/j.scitotenv.2017.12.159

Vilardi G, Sebastiani D, Miliziano S, Verdone N, Di Palma L (2018) Heterogeneous nZVI-induced Fenton oxidation process to enhance biodegradability of excavation by-products. Chem Eng J 335:309-320. https://doi.org/10.1016/j.cej.2017.10.152

Von Sonntag C (2008) Advanced oxidation processes: mechanistic aspects. Water Sci Technol 58:1015-1021. https://doi. org/10.2166/wst.2008.467 
Von Sonntag C, Schuchmann HP (1997) Peroxy radicals in aqueous solutions. In: Alfassi ZB (ed) Peroxy radicals, Wiley, pp 173-234

Vörösmarty CJ, McIntyre PB, Gessner MO, Dudgeon D, Prusevich A, Green P, Glidden S, Bunn SE, Sullivan CA, Liermann CR, Davies PM (2010) Global threats to human water security and river biodiversity. Nature 467:555-561. https://doi.org/10.1038/ nature 09440

Walter MV, Vennes JW (1985) Occurrence of multiple-antibiotic resistant enteric bacteria in domestic sewage and oxidative lagoons. Appl Environ Microbiol 50:930-933

Wan Z, Hu J, Wang J (2016) Removal of sulfamethazine antibiotics using $\mathrm{Ce}-\mathrm{Fe}$-graphene nanocomposite as catalyst by Fenton-like process. J Environ Manag 182:284-291. https://doi. org/10.1016/j.jenvman.2016.07.088

Wang A, Li YY, Estrada AL (2011) Mineralization of antibiotics sulfamethoxazole by photoelectron-Fenton treatment using activated carbon fiber cathode and under UVA radiation. Appl Catal B Environ 102:378-386. https://doi.org/10.1016/j.apcat b.2010.12.007

Wang H, Jiang H, Wang S, She W, He J, Liu H, Huang Y (2014) $\mathrm{Fe}_{3} \mathrm{O}_{4}$-MWCNT magnetic nanocomposites as effi peroxidase mimic catalyst in Fenton-like reaction for water purifi tion without $\mathrm{pH}$ limitation. RSC Adv 4:45809-45815. https:// doi.org/10.1039/C4RA07327D

Wang L, Cao M, Ai Z, Zhang L (2015) Design of a highly efficient and wide $\mathrm{pH}$ electro-Fenton oxidation system with molecular oxygen activated by ferrous-tetrapolyphosphate complex. Environ Sci Technol 49(5):3032-3039. https://doi.org/10.1021/es505984y

Wang N, Zheng T, Zhang G, Wang P (2016) A review on Fenton-like processes for organic wastewater treatment. J Environ Chem Eng 4(1):762-787. https://doi.org/10.1016/j.jece.2015.12.016

Watkinson AJ, Murby EJ, Costanzo SD (2007) Removal of antibiotics in conventional and advanced wastewater treatment: implications for environmental discharge and wastewater recycling. Water Res 41:4164-4176. https://doi.org/10.1016/j.watres.2007.04.005

Weller C, Horn S, Herrmann H (2013a) Effects of Fe(III)-concentration, speciation, excitation-wavelength and light intensity on the quantum yield of iron(III)-oxalato complex photolysis. J Photochem Photobiol A Chem 255:41-49. https://doi.org/10.1016/j. jphotochem.2013.01.014

Weller C, Horn S, Herrmann H (2013b) Photolysis of Fe(III) carboxylato complexes: Fe(II) quantum yields and reaction mechanisms. J Photochem Photobiol A Chem 268:24-36. https://doi. org/10.1016/j.jphotochem.2013.06.022

Werber JR, Osuji CO, Elimelech M (2016) Materials for next-generation desalination and water purifi membranes. Nat Rev Mater 1(5):16018. https://doi.org/10.1038/natrevmats.2016.18

World Health Organization (2017) A report on progress on drinking water: 2017 update and Sustainable Development Goal Baselines

Wu D, Chen Y, Zhang Z, Feng Y, Liu Y, Fan J, Zhang Y (2016) Enhanced oxidation of chloramphenicol by GLDA-driven pyrite induced heterogeneous Fenton like reaction at alkaline condition. Chem Eng J 294:49-57. https://doi.org/10.1016/j. cej.2016.02.097

Xing ZP, Sun DZ (2009) Treatment of antibiotic fermentation wastewater by combined polyferric sulfate coagulation, Fenton and sedimentation process. J Hazard Mater 168:1264-1268. https:// doi.org/10.1016/j.jhazmat.2009.03.008

Yahya MS, Beqqal N, Guessous A, Arhoutane MR, Kacemi KE (2017) Degradation and mineralization of moxifl xacin antibiotic in aqueous medium by electro-Fenton process: kinetic assessment and oxidation product. Cog Chem 3:1290021-1290032. https:// doi.org/10.1080/23312009.2017.1290021

Yalfani MS, Contreras S, Medina F, Sueiras J (2009) Phenol degradation by Fenton's process using catalytic in situ generated hydrogen peroxide. Appl Catal B Environ 89:519-526. https://doi. org/10.1016/j.apcatb.2009.01.007

Yuan Y, Lai B, Tang YY (2016) Combined $\mathrm{Fe}^{0} /$ air and Fenton process for the treatment of dinitrodiazophenol (DDNP) industry wastewater. Chem Eng J 283:1514-1521. https://doi.org/10.1016/j. cej.2015.08.104

Zepp RG, Faust BC, Jürg H (1992) Hydroxyl radical formation in aqueous reactions ( $\mathrm{pH} 3-8)$ of iron(II) with hydrogen peroxide: the photo-Fenton reaction. Environ Sci Technol. https://doi. org/10.1021/es00026a011

Zhang G, Ji S, Xi B (2006) Feasibility study of treatment of amoxicillin wastewater with a combination of extraction, Fenton oxidation, reverse osmosis. Desalination 196:32-42. https://doi. org/10.1016/j.desal.2005.11.018

Zhang Y, He C, Sharma VK, Li X, Tian S, Xiong Y (2011a) A coupling process of membrane separation and heterogeneous Fenton-like catalytic oxidation for treatment of acid orange IIcontaining wastewater. Sep Purif Technol 80:45-51. https://doi. org/10.1016/j.seppur.2011.04.004

Zhang Y, He C, Sharma V, Li X, Tian S, Xiong Y (2011b) A new reactor coupling heterogeneous Fenton-like catalytic oxidation with membrane separation for degradation of organic pollutants. J Chem Technol Biotechnol 86:1488-1494

Zhang W, Gao H, He J, Yang P, Wang D, Ma T, Xia H, Xu X (2017) Removal of norfl xacin using coupled synthesized nano scale zero valent iron (nZVI) with $\mathrm{H}_{2} \mathrm{O}_{2}$ system: optimization of operating conditions and degradation pathway. Sep Purif Technol 172:158-167. https://doi.org/10.1016/j.seppur.2016.08.008

Zubir NA, Yacou C, Zhang X, Diniz da Costa JC (2014a) Optimization of graphene oxide-iron oxide nanocomposite in heterogeneous Fenton like oxidation of acid orange 7. Environ Chem Eng 2:1881-1888. https://doi.org/10.1016/j.jece.2014.08.001

Zubir NA, Yacou C, Motuzas J, Zhang X, Diniz da Costa JC (2014b) Structural and functional investigation of graphene oxide- $\mathrm{Fe}_{3} \mathrm{O}_{4}$ nanocomposites for the heterogeneous Fenton like reaction. Sci Rep 4:1-8. https://doi.org/10.1038/srep04594 